\title{
Pioneer Growth Cone Steering Along a Series of Neuronal and Non-Neuronal Cues of Different Affinities
}

\author{
Michael Caudy* and David Bentley $\dagger$ \\ *Biophysics Group and †Department of Zoology, University of California, Berkeley, California 94720
}

\begin{abstract}
We have analyzed the morphology of over 5000 Til pioneer growth cones labeled with anti-HRP, which reveals the disposition of axons, growth cone branches, and filopodia. Ti1 axon pathways typically consist of a sequence of 7 characteristically oriented segments, with a single, distinct reorientation point between each segment. Growth cones exhibit the same orientations and reorientations in a given region as do axon segments at later stages. The single, distinct reorientations suggest that growth cones make discrete switches between guidance cues as they grow.
\end{abstract}

Til growth cones are guided by various types of cues. A set of 3 immature identified neurons serves as nonadjacent guidepost cells and lies at the proximal end of 3 of the axon segments. To form another segment, growth cones reorient along a limb segment boundary within the epithelium. Growth cones also respond consistently to, and orient toward, a specific mesodermal cell, which may be a muscle pioneer. Thus, growth cones respond to at least 3 different types of cells in the leg.

Ti1 growth cones exhibit a hierarchy of affinity for these cues. Guidepost neurons are the dominant cues in that contact with them reorients growth cones from guidance by the other types of cues.

Growth cone branches are exclusively oriented to specific cues. Growth cones reorient by extending a branch directly to the cue of highest affinity and by withdrawing any branches that are extended to a cue of lesser affinity. A single filopodium in direct contact with a guidepost neuron can reorient a growth cone that still has multiple filopodia or even prominent branches specifically oriented to a previous cue of lesser affinity. These observations suggest that growth cone steering may not result simply from passive adhesion and filopodial traction, but may involve more active processes.

During the course of development, axons grow along specific pathways to their appropriate targets. Many axons are guided by fasciculating with previously formed axons and nerves. However, the initial axons growing through any region must "pioneer" a pathway through an axonless environment, and therefore must be guided by other types of cues. We are investigating the guidance mechanisms for afferent pioneer axons in the legs of grasshopper embryos.

Bate (1976) identified the first peripheral axons to arise in embryonic legs and antennae of grasshoppers as those of pairs of neurons located at the tips of those appendages. In legs, these

\footnotetext{
Received Sept. 13, 1985; revised Dec. 6, 1985; accepted Dec. 18, 1985.

We thank Alma Toroian-Raymond for light-microscope histology and technical assistance, and Dr. Marty Shankland for critiquing the manuscript. Support was provided by NIH NS09074-14 and NIH 2T32-GM07379. D.B. was also supported by an NIH Jacob Javits Award, and M.C. by an Einstein Fellowship for Developmental Neuroscience, from the University of California.

Correspondence should be addressed to Michael Caudy, Department of Physiology, University of California, San Francisco, CA 94143

Copyright $(1986$ Society for Neuroscience $0270-6474 / 86 / 061781-15 \$ 02.00 / 0$
}

(Ti1) pioneer axons extend proximally from their cell bodies, and then turn and grow circumferentially before making a second turn and continuing proximally to the CNS (Bentley and Keshishian, 1982a, b; Ho and Goodman, 1982).

Along their route, the Til axons extend between a set of putative guidepost neurons, with which they selectively dyecouple. Of these neurons, the $\operatorname{Tr} 1$ neuron and (pair of) $\mathrm{Cx} 1$ neurons are located at the characteristic turns on the route (Bentley and Keshishian, 1982a, b; Ho and Goodman, 1982; Keshishian and Bentley, 1983a-c). Selective ablation of the $\mathrm{Cx} 1$ guidepost cells has shown them to be necessary for normal pathfinding (Bentley and Caudy, 1983a, b). Another cue for Til growth cones is a limb segment boundary, which orients them circumferentially (Bentley and Caudy, 1983b; Caudy and Bentley, 1986). There also is evidence that a proximal increase in epithelial affinity within segments can guide Til growth cones proximally when they are not in contact with higher affinity cues (Caudy and Bentley, 1986).

Here we describe the detailed route taken by the Til growth cones in normal embryos, and their characteristic interactions with identified guidepost neurons and other extrinsic cues. These interactions, and the detailed dispositions of growth cone branches and filopodia, suggest that the Til growth cones have different affinities for their different cues. These interactions and dispositions also are interpreted with regard to their implications for models of growth cone steering based on adhesionmediated (Bray 1979, 1982; Letourneau, 1975a, b, 1979, 1983); or active (Cooper and Schliwa, 1985) responses to guidance cues.

\section{Materials and Methods}

\section{Antibody labeling}

The anti-HRP labeling protocol is described in detail in Caudy and Bentley (1986). In brief, it consists of the following steps: (1) fixation in $4 \%$ formaldehyde in physiological saline; (2) $1 \mathrm{hr}$ rinse in PBS with Triton ("rinse"); (3) 12-24 hr incubation in $0.02 \%$ rabbit anti-HRP serum; (4) $1 \mathrm{hr}$ wash in rinse with $1 \% \mathrm{BSA}$; (5) $12-24 \mathrm{hr}$ incubation in $0.04 \%$ TRITC-conjugated goat anti-HRP (IgG fraction); (6) $1 \mathrm{hr}$ wash in rinse with $1 \% \mathrm{BSA}$; and (7) whole-mount under coverglass with 40 $\mu \mathrm{m}$ wire spacers between coverglass and slides in 1:9 PBS: glycerol mountant with antioxidants. Using this labeling technique, we have examined the morphologies of approximately $5000 \mathrm{Til}$ growth cones ( $2 \mathrm{Til} / \mathrm{s} / \mathrm{leg}$ ) in approximately 500 animals from about 50 different clutches of eggs.

\section{Terms}

Filopodia usually can be clearly distinguished from branches. Filopodia are the smallest diameter processes observed $(<0.5 \mu \mathrm{m})$ and typically are very uniform in diameter from their tip to within 2 or $3 \mu \mathrm{m}$ of their base. Filopodial clusters are several filopodia extended in a highly oriented group with few or no filopodia extended within the adjacent regions. Branches of growth cones are any processes larger than a filopodium or smaller than an axon. They can vary widely in diameterfrom 0.5 to about $5 \mu \mathrm{m}$-and also can form branches of their own. 
Table 1. Mean length of, and guidance cues for, the characteristic Til axon segments ${ }^{a}$

\begin{tabular}{|c|c|c|c|}
\hline Segment & & $\begin{array}{l}\text { Mean length } \\
(\mu \mathrm{m} \pm \mathrm{SD})\end{array}$ & Known or putative cues \\
\hline$a$ & $=$ & $37.7 \pm 16.1$ & $\begin{array}{l}\text { 1. Intrinsic polarity of the Til cell } \\
\text { bodies } \\
\text { 2. Proximal increase in epithelial } \\
\text { affinity }\end{array}$ \\
\hline$b$ & $=$ & $54.5 \pm 12.1$ & Fel guidepost neuron \\
\hline$c$ & $=$ & $27.6 \pm 10.1$ & $\begin{array}{l}\text { 1. Proximodorsal orienting factor } \\
\text { 2. } m 1 \text { mesodermal cell (probable } \\
\text { muscle pioneer }{ }^{b} \text { ) } \\
\text { 3. Other dorsally located cells, e.g., } \\
m 2 \\
\text { 4. Femur-trochanter segment } \\
\text { boundary }\end{array}$ \\
\hline$d$ & $=$ & $55.7 \pm 6.1$ & $\operatorname{Trl}$ guidepost neuron \\
\hline$e$ & $=$ & $38.2+19.2$ & Distal segment boundary of coxa \\
\hline$f$ & $=$ & $73.7 \pm 21.7$ & Cxl guidepost neurons \\
\hline$g$ & $=$ & $127.6 \pm 23.8$ & $\begin{array}{l}\text { 1. Unidentified, strong, proximal } \\
\text { orientation factor } \\
\text { 2. } p \text { cells }\end{array}$ \\
\hline
\end{tabular}

Total $=416.4$

$a, b: n=10 ; c-g: n=11$

a Segments are depicted in Figure 10.

${ }^{h}$ See text; cell depicted in Figure $5 \mathcal{A}$ (Ball et al., 1985).

c Total of mean segment lengths traversed by Til growth cones.

\section{Measurement of axon segments}

The length of the $a$ segment (Fig. 10) is measured as the distance between the point where the Til axons emerge from their cell bodies to the point where they reorient toward the Fel cell. The length of the $b$ segment is the distance between the reorientation toward the Fel cell to the point of contact with that cell, plus the length of axon running along the Fel cell to the point where the axon leaves it. The remaining axon segments are measured similarly.

\section{Definition of cells, axes, and stages of development}

The names of the various cells and limb axes are defined in the Materials and Methods section of Caudy and Bentley (1986), as are the stages of neuronal differentiation. Gencral staging of embryogencsis is described in Bentley et al. (1979).

\section{Results}

\section{Characteristic Til Axon and Growth Cone Morphologies}

Til axons closely follow the initial growth cone routes

Til axons are not displaced laterally, or "dragged" (Gunderson and Park, 1984), over the epithelial substrate by the motile growth cone. Distinct axon reorientations are regularly observed (Figs. 1, $B, D, 2, A, B, 3 C$ ), indicating that the axons are firmly attached to the substrate, at least at the point of reorientation. In some cases, axon reorientations are observed immediately behind the growth cone (Fig. $3 \mathrm{C}$ ), suggesting that the axons are attached to the substrate either as they are laid down, or very soon thereafter. In addition, $\mathrm{Ti} 1$ axons generally have distinct edges flattened against the epithelial substrate at all points along their length, an indication that they are attached all along their length.

Distinct reorientations occur at characteristic locations (see
Table 2. Percentage of Til guidance by guidepost neurons between each pair of neurons on the path

\begin{tabular}{ll} 
Neuron pair & Til guidance (\%) \\
\hline From Til to Fel & 59 \\
From Fel to $\mathrm{Trl}$ & 67 \\
From $\mathrm{Trl}$ to $\mathrm{Cx} 1 \mathrm{~s}$ & 66
\end{tabular}

below), and are observed at the same location both soon after the growth cone reorients and at substantially later stages. In Figure $1, B$ and $D$, a reorientation that is frequently observed about $30-50 \mu \mathrm{m}$ proximal to the Til cell bodies is present both when the growth cone is at the Fel cell and about $6 \mathrm{hr}$ later when the growth cone is at the Tr1 cell. These observations indicate that the distinct reorientations observed in axon morphologies accurately reflect the location and direction of reorientations made by growth cones at earlier stages. Therefore, the morphologies of recently established, or "nascent," axons can be used to determine the precise routes taken by growth cones in a particular leg.

\section{Til axons follow a stereotyped route consisting of distinctly oriented segments}

Observation of growth cone and nascent axon morphologies at various points along the path has shown that the overall Til axon morphology consists of distinct, highly stereotyped segments of axon. In normal pathways, a series of straight, characteristically oriented segments of axon is observed (Figs. 1, $B, D, 2,3$, and 7 ). In the most complex casc of such normal pathways, the overall path is comprised of 7 distinct axon segments, which are schematized and labeled in Figure 10. The average lengths of these axon segments are given in Table 1 (see also Table 2). We will describe the most complex case first; variants in which certain segments are missing will be discussed later.

Segment $a$ : The segment of initial outgrowth of the pair of Til axons from their cell bodies is oriented proximally along the geometric axis of the tibia (Fig. $1, A, B, D$; schematized in Fig. 10).

Segment $b$ : The next segment is initiated by a single, distinct reorientation and is oriented in the proximoventral direction (Fig. 1, $B, D$ ). This segment extends straight in this direction to the Fel identified neuron and then extends along the dorsal edge of that neuron (Fig. 1D). The segment is terminated where the axons leave the proximal edge of the Fel cell and distinctly reorient (Fig. $1, C, D$ ).

Segment $c$ : On leaving the Fel neuron, Til axons (Figs. $1 D$, $2 A$ ) and growth cones (Figs. $1, B, C, 5 A, 6 A$ ) again distinctly reorient, this time in the proximodorsal direction. This reorientation is more acute than is necessary to orient proximally along the geometric axis of the limb (cf. drawing in Fig. 10), so that the $c$ segment always is oriented dorsal to the proximally located Tr1 neuron (Fig. 1, C, D).

Segment $d$ : This segment begins with a distinct reorientation from the $c$ segment and is oriented proximoventrally (Figs. $1 D$; $2, A, B)$. The $d$ segment extends directly to the $\operatorname{Tr} 1$ neuron and then along that neuron to its ventral edge (Figs. $4 D, 5 D$ ).

Segment $e$ : This segment is oriented circumferentially from its origin at the $\operatorname{Trl}$ cell (Figs. $2 B, 3,5 D$; see also Bentley and Caudy, 1983b). It extends straight ventrally and, as a result, is oriented toward a point that lies about $20 \mu \mathrm{m}$ straight distal to the pair of $C x 1$ identified neurons (Fig. $3 C$ ). The $e$ segment terminates with a distinct reorientation toward the $\mathrm{Cx} l$ guidepost neurons.

Segment $f$ : This segment is oriented toward the Cx1 neurons 

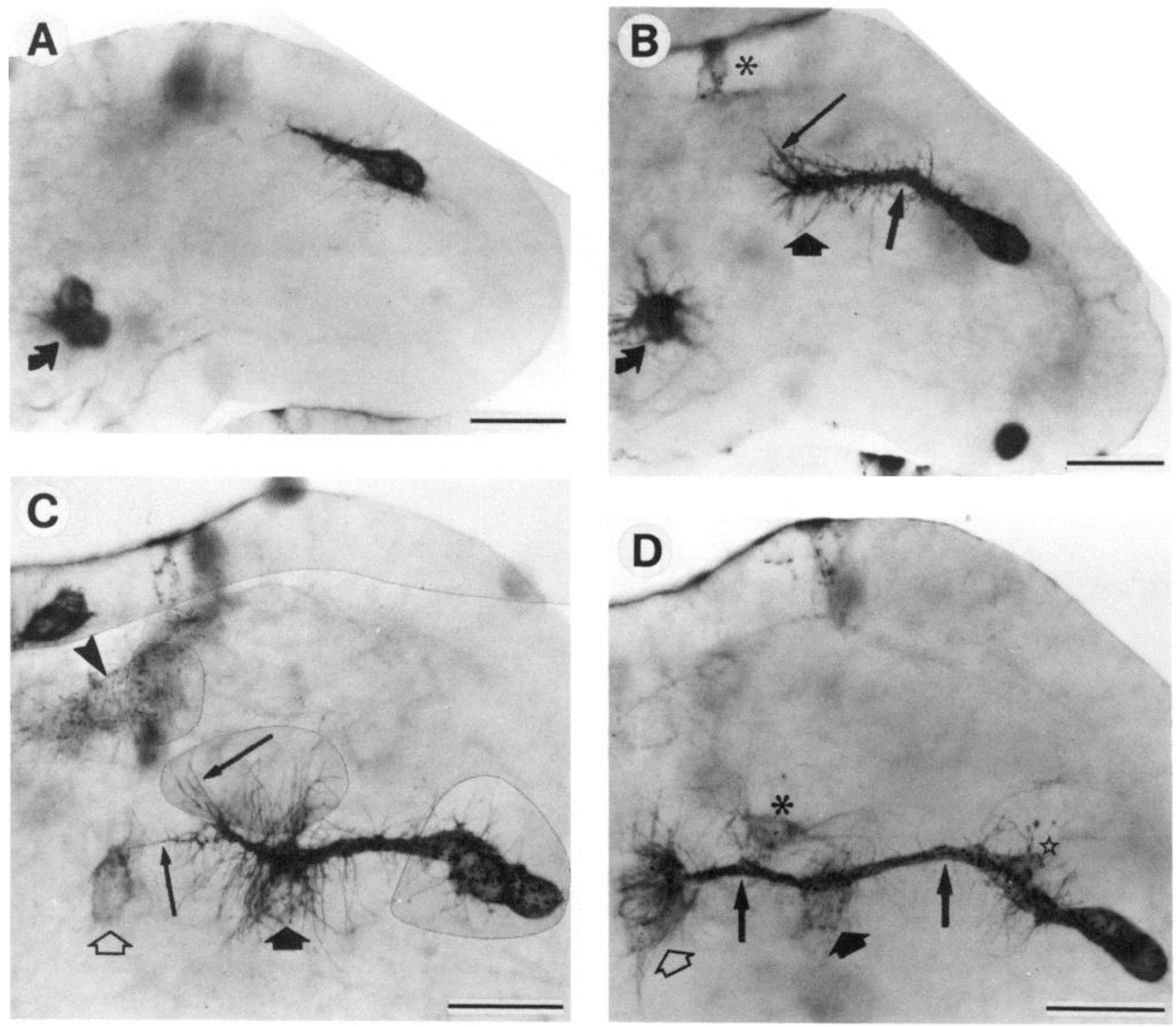

Figure 1. Til morphologies along the $a, b, c$, and $d$ segments of the limb. $A$, The pair of Til neurons has begun proximal axonogenesis, and their axons are extended straight proximally along the geometric axis of the limb. This forms the $a$ segment (see text). The Til growth cones typically have no branches on the $a$ segment. The Til cell bodies also are aligned along the proximodistal axis of the leg. Curved arrow, Cx1 cells. Calibration bar, $50 \mu \mathrm{m}$. B. The Til cells are aligned along the limb axis and have extended axons proximally along the $a$ segment. The axons distinctly reorient at a point (upward arrow) about $40 \mu \mathrm{m}$ proximal to the Til cell bodies and then extend directly to the site (arrowhead) where the Fel cell arises. This forms the $b$ segment (see text). From the Fel site, 2 clusters of filopodia (downward arrow) are extended proximodorsally in the direction of a pair of anti-HRP labeled cells (asterisk) in the epithelium. Curved arrow, Cxl cells. Calibration bar, $50 \mu \mathrm{m}$. $C$, The Til axons have extended proximally from their cell bodies and have contacted the Fel cell (solid arrowhead). Growth cones have numerous lateral filopodia, about 25-35 $\mu \mathrm{m}$ in length, and have extended proximodorsally from the Fel cell, along the $c$ segment (see text). A cluster of leading filopodia (downward arrow) is extended directly toward the large, lamellar $m l$ cell (caret), which is labeled with anti-HRP. From the same growth cone, a branch is extended proximoventrally along a single filopodium (upward arrow) that is in direct contact with a labeled Trl cell (open arrowhead). Calibration bar, $50 \mu \mathrm{m}$. D. The Til axons have extended proximodorsally from the Fel cell (solid arrowhead) to a point (upward arrow at left) from which they have reoriented proximoventrally and extended directly toward the Trl cell (open arrowhead). This forms the $d$ segment (see text). The Til growth cones are spreading over the $\operatorname{Tr} 1$ neuron, which has begun axonogenesis in the ventral direction, along the distal segment boundary of the coxa. A cell (asterisk) labeled with anti-HRP lies near the point of reorientation, but is not precisely at it. A lamellum (star) is extended lateral to the Til axons where they have crossed the tibia-femur segment boundary (see Caudy and Bentley, 1986). The Til axons also make a distinct reorientation (arrow at right) toward the Fel cell after initially growing proximally along the $a$ segment. Thus, in this leg the characteristic $a, b, c$, and $d$ segments all are exhibited in the axon morphology of a growth cone that has extended from the Til cell bodies to the $\operatorname{Tr} 1$ neurons. Calibration bar, $50 \mu \mathrm{m}$.

(Fig. 3C), extends directly to and along them, and ends at their proximal edge.

Segment $g$ : This segment begins at the proximal edge of the $\mathrm{Cxl}$ neurons, extends straight proximally to the CNS without further reorientations (Figs. $4 A, 7 B$ ), and ends with contact with the CNS.
In summary, in normal embryos the Til axons are oriented in a characteristic direction at each point along their route to the CNS. Furthermore, since axon orientations reflect the orientation that the growth cone had as it grew, the growth cones apparently were specifically oriented at all points along the path, and thus apparently did not grow randomly at any point. Fur- 

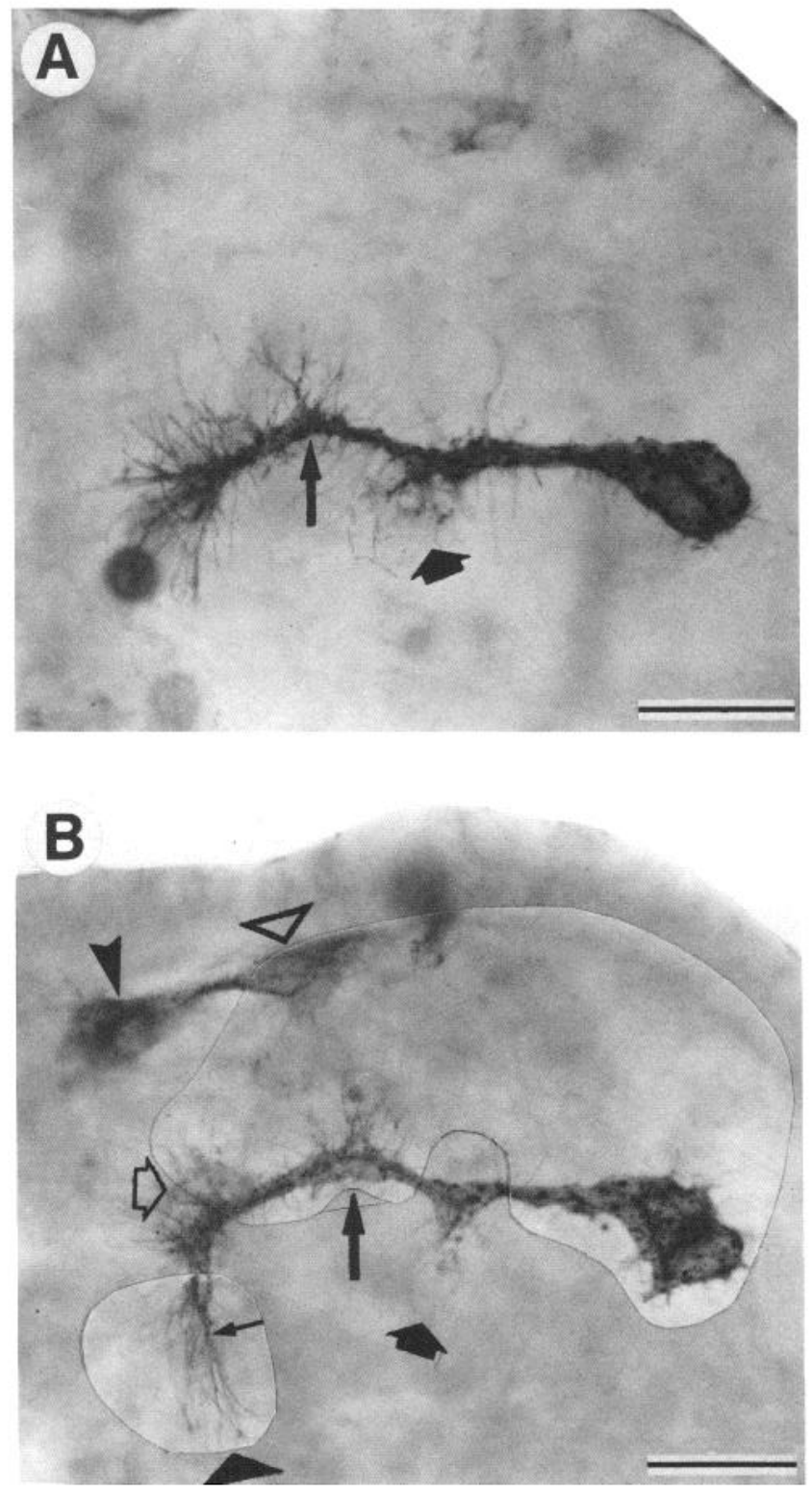

Figure 2. Til morphologies along the $c, d$, and $e$ segments. $A$, The Til axons have extended straight from their cell bodies to contact a faintly labeled Fel cell (solid arrowhead). The Til axons have their characteristic proximodorsal orientation along the $c$ segment, to a point (arrow) where they distinctly reorient and extend directly to the Trl cell site, to form the $d$ segment. A single, small branch also extends dorsally from the reorientation point (arrow). Calibration bar, $50 \mu \mathrm{m}$. B. The Til axons have extended straight from their cell bodies to a point from which lateral processes have contacted a faintly labeled Fel cell (solid arrowhead). From that point they extend along the $c$ segment in the direction of the dorsally located $m l$ cell (carat), which has labeled with anti-HRP in this leg ( $c f$. Figs. $1 C$ and $6 C$ ). The proximodorsal orientation is maintained to the point (arrow) where the Til axons distinctly reorient and extend directly along the $d$ segment to a faintly labeled Trl cell (open arrowhead). The $\operatorname{Tr} 1$ cell lies on the distal segment boundary of the coxa. After contacting that cell, the Til growth cones have reoriented circumferentially along the segment boundary (solid triangle), to begin forming the $e$ segment (see text). As in $A$, Til processes (several filopodia) extend straight dorsally from the point of reorientation (arrow). In this leg, a second dorsally located cell, $m 2$ (open triangle) has labeled with anti-HRP. A broad lamellar process is extended ventrally from the second dorsal cell. Calibration bar, $50 \mu \mathrm{m}$.
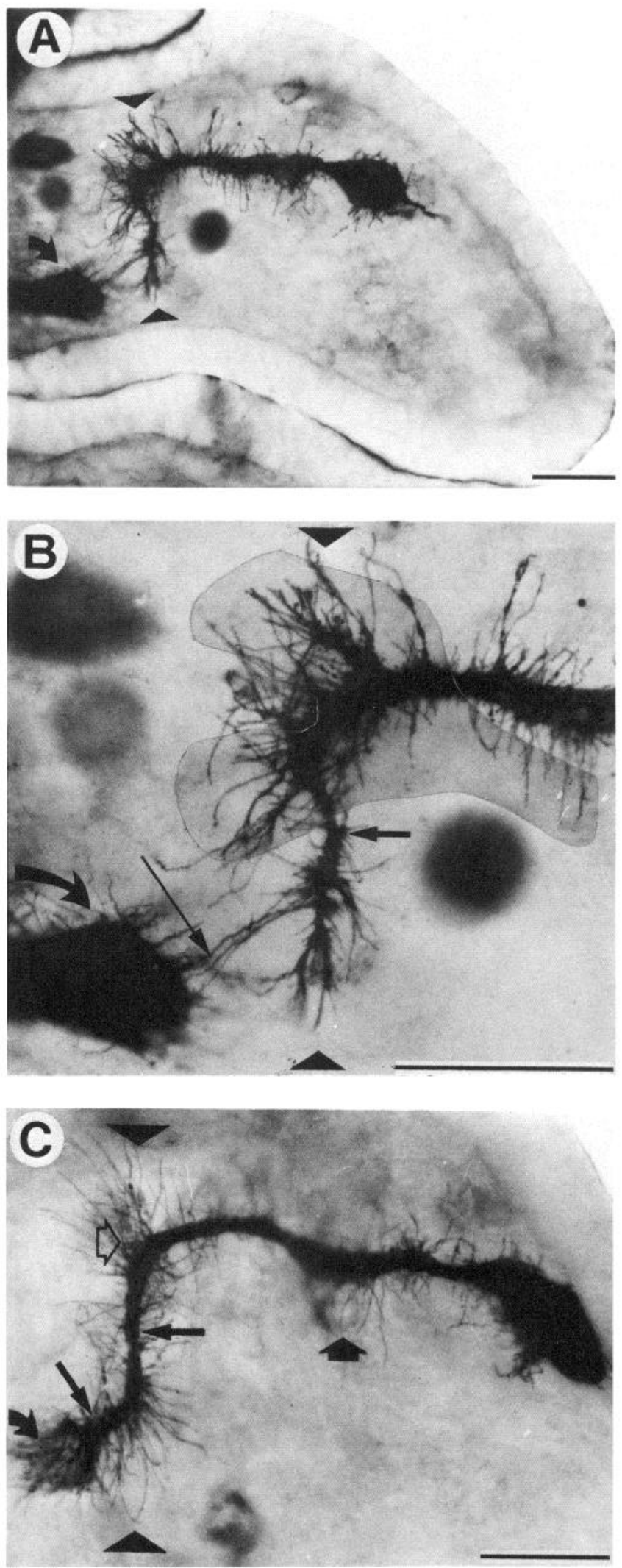

Figure 3. Growth cone morphologies along the $e$ and $f$ segments. $A$, The Til axons extend proximally to the distal segment boundary of the coxa (solid triangles), where their growth cones abruptly reorient and extend ventrally along the segment boundary. Curved arrow, $\mathrm{Cx} 1$ cells. Calibration bar, $50 \mu \mathrm{m}$. B, The same leg at higher magnification. Til growth cones extend along the segment boundary to form segment $e$ 

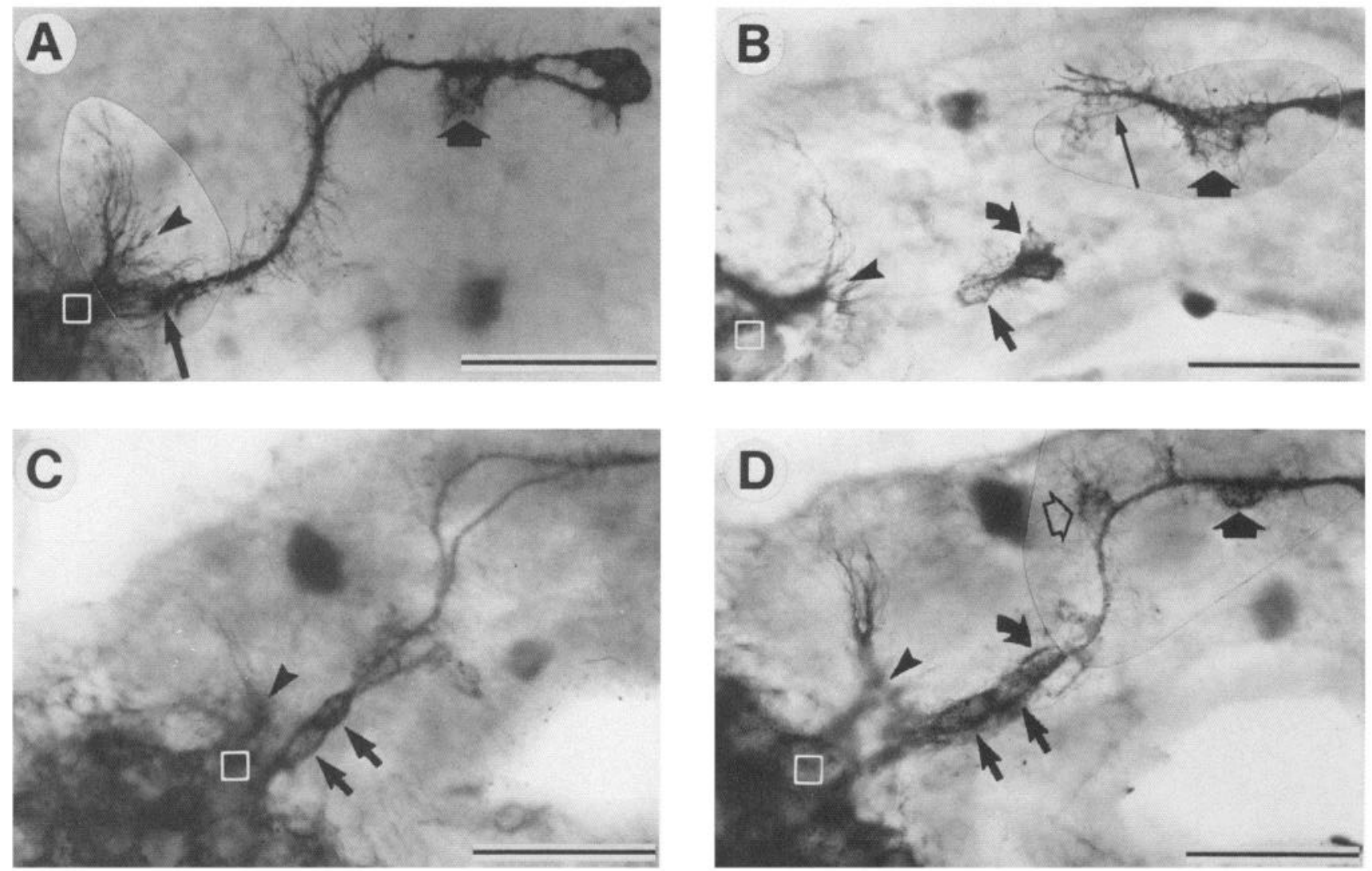

Figure 4. Til morphologies and anti-HRP labeled cells along the $g$ segment. $A$, The Til growth cones (arrow) have nearly reached the boundary of the CNS (white square). The Til axons are straight and unbranched along the $g$ segment (i.e., the $60-70 \mu \mathrm{m}$ region straight distal to the growth cones), which lies between the Cx1 cells (unlabeled in this leg, but see Figs. 7 and 9) and the CNS. Caret, Efferent processes; solid arrowhead, Fe1 cell. Calibration bar, $100 \mu \mathrm{m}$. B, The pair of Cx1 guidepost cells (curved arrow) has begun axonogenesis before the Til growth cones have reached them. In this leg, another proximal $(p)$ cell (straight arrow) on the final path has labeled with anti-HRP. This $p$ cell lies about $15 \mu \mathrm{m}$ straight proximal to the $\mathrm{Cx} 1$ cells. The Til growth cones have branches extended along the $c$ segment. A single, very thin branch (upward, narrow arrow) is in contact with a labeled Tr1 neuron (not marked). White square, Boundary of CNS; caret, efferent fibers; solid arrowhead, Fe1. Calibration bar, $100 \mu \mathrm{m}$. $C$, The Til axons have reached the CNS boundary (white square). Two adjacent $p$ cells (arrows) have labeled with anti-HRP. The more proximal of these cells is within one cell diameter of the CNS. The more distal of these $p$ cells lies about 20-30 $\mu$ m proximal to the site where the $\mathrm{Cx} 1$ cells are located. Caret, Efferent fibers. Calibration bar, $100 \mu \mathrm{m}$. D, The Til axons have extended proximally to contact the Fel cell (solid arrowhead) and the $\operatorname{Tr} 1$ cell (open arrowhead), and have then turned circumferentially along the distal coxal segment boundary. The axons have turned proximally from the segment boundary and extended to the Cxl cells (curved arrow). Two additional p cells (straight arrows) lie straight proximal to the Cx1 cells, so that together these cells form a continuous chain along the final path that reaches nearly to the CNS (white square). Caret, Efferent fibers. Calibration bar, $100 \mu \mathrm{m}$.

thermore, since single, distinct reorientations typically initiate the characteristic segments, it would appear that at these points the growth cones made a discrete switch in the guidance cue that they were following. We will therefore consider these characteristic segments of the path with regard to known or possible guidance cues.

\section{Growth cone interactions with guidepost neurons}

The best characterized guidance cues for the Til growth cones are identified guidepost neurons. In normal embryos the Til

(horizontal arrow). Three filopodia (downward arrow) are in direct contact with the Cxl neurons (curved arrow). Calibration bar, $50 \mu \mathrm{m}$. $C$, The Til axons extend proximally to the Fel neuron (solid arrowhead) and then to the $\operatorname{Tr} 1$ neuron (open arrowhead). At the Trl neuron, they reorient circumferentially along the distal segment boundary of the coxa (solid triangles) to form segment $e$. They then make a single, distinct reorientation toward the $\mathrm{Cx} 1$ neurons (curved arrow) and extend directly to them to form the characteristic $f$ segment (downward arrow). The Til growth cones are at the $\mathrm{Cx} 1$ neurons and are wrapping branches and filopodia around one of them. Calibration bar, $50 \mu \mathrm{m}$. axons make direct contact with these cells and selectively dyecouple with them (Bentley and Keshishian, 1982a, b; Keshishian and Bentley, 1983a; Taghert et al., 1982). Deletion of the Cx1 guidepost cells has shown them to be a necessary cue for guidance along the normal path (Bentley and Caudy, 1983a, b).

\section{Axon reorientations toward guidepost neurons}

Three of the characteristic Til axon segments $-b, d$, and $f$-are each oriented toward, make direct contact with, and then extend along the Fe1, Tr1, and $\mathrm{Cx} 1$ guidepost neurons, respectively. The point at which the Til axons leave a guidepost neuron, after extending along it, typically is the precise point where the axons make a distinct reorientation onto the next characteristic axon segment (Figs. 1, $C, D, 5 D$ ). Thus, that point of reorientation clearly defines the termination of the previous axon segment.

\section{Growth cone branches and filopodia selectively wrap guidepost neurons}

Growth cones do not simply extend along guidepost neurons that they have contacted. Frequently growth cones also profusely wrap labeled guidepost neurons with filopodia (Fig. 1, $C, D, 3 C$ ) 

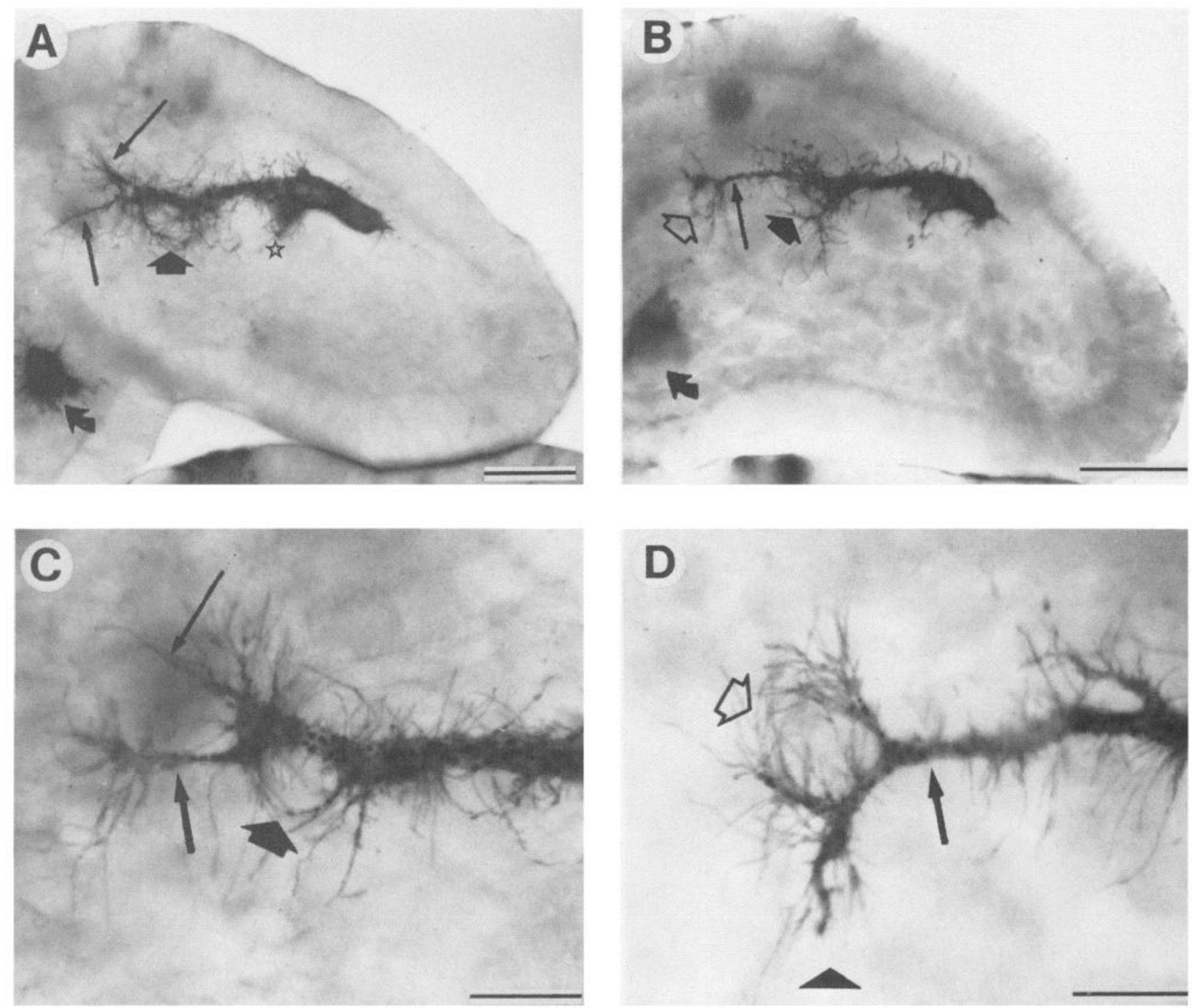

Figure 5. Selective wrapping of unlabeled Fel and Trl guidepost cells by Til branches and filopodia. $A$, The Til axons have extended proximally from their cell bodies and have extensively wrapped an unlabeled Fel cell (solid arrowhead) with branches and filopodia. Two branches have extended beyond the Fel cell: one (upward arrow) directly toward the site where the Trl cell arises, and one (downward arrow) proximodorsally along the characteristic $c$ segment toward the region where the $m 1$ cell occasionally labels with anti-HRP (see Figs. $1 C$ and $6, C, D$ ). A prominent, triangular-shaped lamellum (star) is extended laterally from the Til axons, where they have crossed the tibia-femur segment boundary (see Caudy and Bentley, 1986). Curved arrow, Cx1 cells. Calibration bar, $50 \mu \mathrm{m}$. B. The Til axons have extended proximally and have wrapped an unlabeled Fel cell (solid arrowhead) with branches and filopodia. At least one Til axon (arrow) has extended further proximally and is wrapping an unlabeled Trl cell (open arrowhead) with branches and filopodia. Curved arrow, Cxl cells. Calibration bar, $50 \mu \mathrm{m}$. C, The Til axons have wrapped an unlabeled Fel cell (solid arrowhead) with branches and filopodia, which extend around the complete circumference of the cell on the inner epithelial surface. Two Til processes have extended beyond the Fe1 cell: a prominent branch (upward arrow) is extended directly to the site where the Tr1 cell arises, and several filopodia (downward arrow) are extended proximodorsally toward the region where the $m l$ cells are typically located (see Figs. $1 C, 6, B, C, D$ ). Calibration bar, $25 \mu \mathrm{m}$. $D$, The Til axons (arrow) have extended proximally and have contacted an unlabeled Trl cell (open arrowhead). Branches and filopodia have wrapped in both directions around the cell on the inner epithelial surface (the plane of focus), and additional filopodia are curving over its surface. The Trl cell lies on the distal boundary of the coxal segment (solid triangle) and a branch is extended ventrally along the segment boundary. Calibration bar, $25 \mu \mathrm{m}$.

and occasionally spread lamellae over them (Fig. $1 D$ ). Branches also are observed to wrap over labeled guidepost neurons and to extend around the circumference of the cell where it contacts the epithelial substrate (Figs. 3, 4B).

The characteristic wrapping of filopodia and branches around neurons frequently reveals the presence and location of unlabeled guidepost neurons (Fig. 5). Filopodia selectively wrap single unlabeled cells at the Fel site (Figs. 5, $A-C, 6 A$ ), the Tr1 cell site (Fig. 5, B, D) and at the site of the Cx1 cells (not shown). Branches extended around the circumference of unlabeled cells also are observed at the $\mathrm{Fe} 1$ site (Figs. $2 A, 5, A-C, 6 C$ ), the $\operatorname{Tr} 1$ cell site (Fig. 5, B, D), and the site of the Cx1 cells (not shown). The selective wrapping of Til growth cone filopodia and branches around guidepost neurons suggests that growth cones specifically respond to contact with these cells. Furthermore, these characteristics allow Til morphologies to be used as indicators of the presence of unlabeled guidepost cells on the path.

\section{Ti1 morphologies and putative cues for the other characteristic segments}

In any given embryo, Til growth cones extending along the characteristic segments that are not oriented toward guidepost 

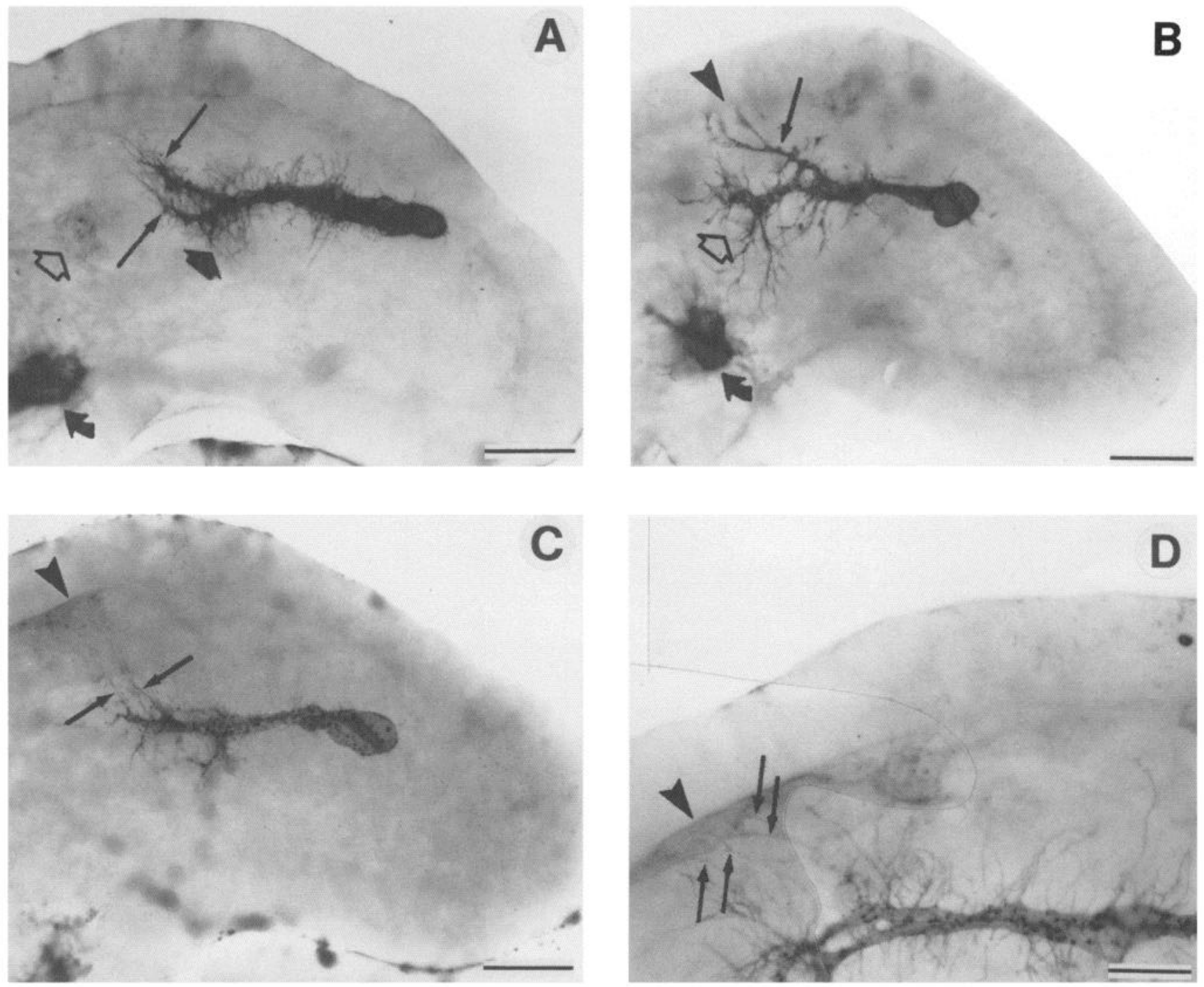

Figure 6. Til growth cone orientation along the $c$ segment. A, The Til axons have contacted an unlabeled Fel cell (solid arrowhead) and wrapped it with filopodia. At the Fel cell site, the pair of Til growth cones has separated (long arrows), and each has independently oriented proximodorsally along a cluster of leading filopodia. The Trl cell (open arrowhead) has begun to label with anti-HRP and lies straight proximal to the Fel cell, but the Til growth cones are oriented toward a region dorsal to the Trl cell, along the characteristic $c$ segment. Curved arrow, Cx 1 cells. Calibration bar, $50 \mu \mathrm{m}$. B. The Til axons have extended proximally to the $\operatorname{Tr} 1$ cell site (open arrowhead) and are wrapping branches around an unlabeled cell at that site. A prominent branch (downward arrow) also has extended proximodorsally from the Fel cell site and has divided into 3 smaller branches that converge at the site of the $m 1$ cell (caret; $c f$. Fig. $1 C$ ). Curved arrow, Cxl cells. Calibration bar, $50 \mu \mathrm{m}$. C, The Til axons have extended proximally from their cell bodies. Several filopodia (arrows) are extended parallel to one another from different sites on the Til growth cone. They are extended proximodorsally toward the $m l$ cell (carat), which is apposed to the inner epithelial surface. Anti-HRP-labeled particles extend radially from the $m l$ cell and may reflect lamellar processes ( $c f$. Fig. $1 C$ ), which the Til filopodia may contact. Calibration bar, $50 \mu \mathrm{m}$. $D$, In another leg, the $m l$ cell (caret) has again labeled with anti-HRP, and several filopodia (arrows) extended proximodorsally from the Til axons have made direct contact with it. At least 2 of the filopodia divide in 2 very near the point where they contact the $m l$ cell. Calibration bar, $25 \mu \mathrm{m}$.

neurons are as highly oriented as growth cones growing toward guidepost cells. Growth along each of the "nonguidepost" segments is initiated by a single distinct orientation (segment $a$ ) or reorientation, as though each resulted from a separate guidance cue. However, growth cones on these segments do not appear to be oriented by a specific, single cell at the termination point.

\section{Segment a: proximal outgrowth from the}

Til cell bodies

Til growth cones and axons do not exhibit filopodial wrapping with labeled or unlabeled cells at the termination point of the $a$ segment (Fig. 1, $A, B$ ). Also, the $a$ segment varies in length by a greater amount than do the $b$ and $d$ segments (Table 1). These observations suggest that the $a$ segment is not oriented by a specific cell or feature at a fixed distance from the Til cell bodies.

The $a$ segment generally is oriented along the geometrical axis of the tibia, in which the Til neurons arise (schematized in Fig. 10). The pair of Til cell bodies are usually aligned along this axis (Figs. 1, 5, $A, B$, and $6 A$ ), so that their axons emerge from the proximal poles of the cell bodies and extend straight proximally until they reorient toward the Fel neuron (Fig. 1, $A, B$, $D$ ). Therefore, proximal axonogenesis along the $a$ segment may be due to internal polarity of the Til cell bodies. Alternatively (or in addition), there also is evidence that proximal guidance of the Til growth cones in this region may result from proximal increases in the affinity of the substrate epithelium for growth cones (Caudy and Bentley, 1986). 

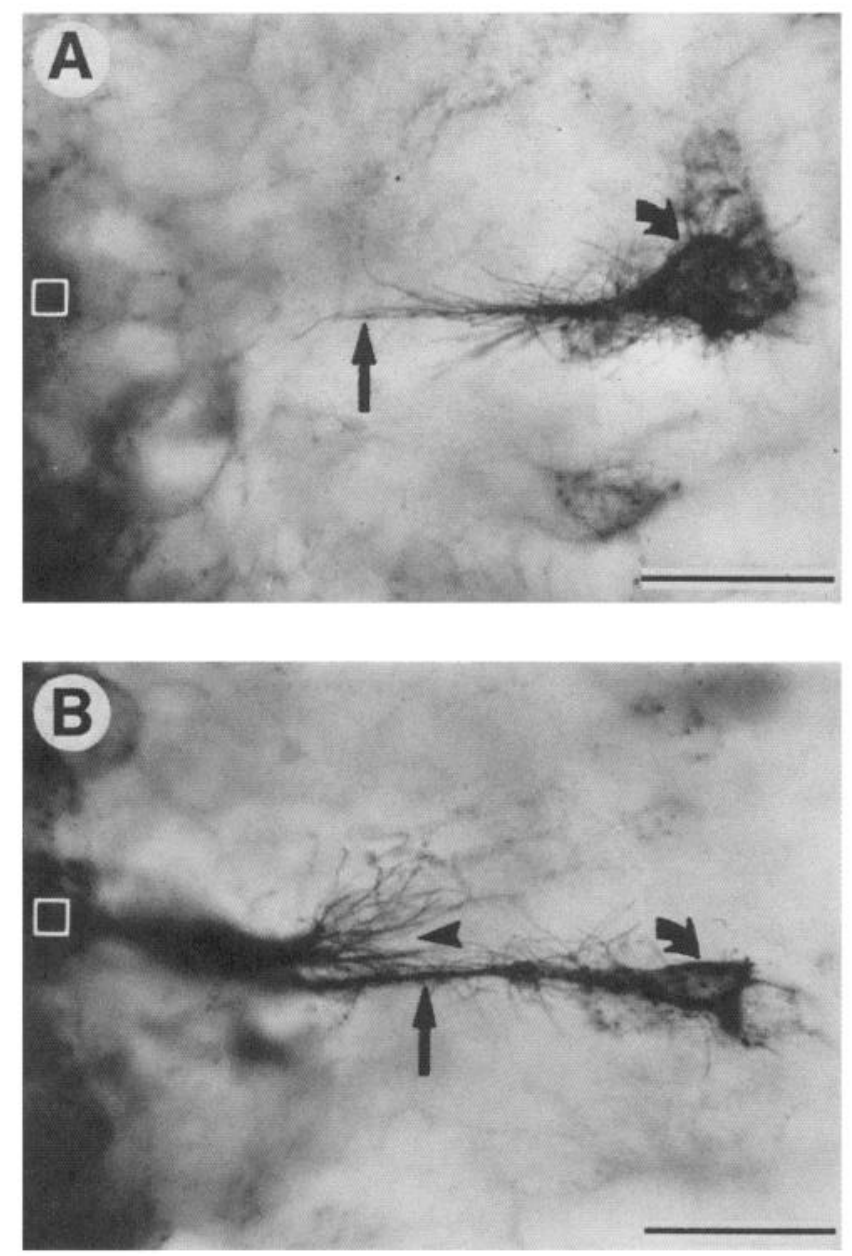

Figure 7. Cxl growth cone and axon morphologies in the $g$ segment region. $A$, In some legs, the $\mathrm{Cx} 1$ neurons (curved arrow) pioneer the same pathway to the CNS (white square) that forms the $g$ segment of the Til axons. When the $\mathrm{Cx} 1$ growth cones extend proximally along this path, their growth cones are highly oriented straight proximally, even to the degree that filopodia tend to lie in very tight clusters (arrow). Calibration bar, $50 \mu \mathrm{m}$. B, At a later stage, when the Cx1 (curved arrow) growth cones have reached the CNS (white square), their axons (arrow) are straight. The $\mathrm{Cx} 1$ axons have no lateral branches and very few lateral filopodia. Caret, Efferent fibers. Calibration bar, $50 \mu \mathrm{m}$.

\section{Segment c: proximodorsal reorientation \\ from the Fel neuron}

The $c$ segment typically is initiated by a single distinct reorientation, suggesting a discrete switch in guidance cue. However, growth cones and axons on the $c$ segment do not exhibit any specific interactions with labeled or unlabeled cells at the termination point of the segment. Furthermore, separated Til growth cones (Fig. 6A) and filopodia (Fig. $6 \mathrm{C}$ ) can orient proximodorsally from different points and extend roughly parallel. They are not oriented toward a single point in the region where subsequent reorientations typically occur. In addition, branches regularly extend proximodorsally beyond the point of reorientation toward $\operatorname{Tr} 1$ (Fig. 8). Also, branches occasionally (although very rarely) extend beyond the typical reorientation point and continue proximodorsally to the dorsal edge of the leg, where they appear to converge on an unlabeled cell (Fig. 6B).

In some legs, a specific cell, $m 1$, transiently labels with antiHRP in this same region at the dorsal edge of the leg (Figs. $1 C$, 6, $C, D$ ). Anti-HRP antibodies typically are specific for a cellsurface molecule on insect neurons (Jan and Jan, 1982), although some other cell types also label (Jan and Jan, 1982; Shankland and Bentley, 1983). However, the $m l$ cell does not appear to be a neuron. Unlike known neurons, it only occasionally labels with anti-HRP. Furthermore, even when it does label, it apparently does so only transiently, as labeled $m l$ cells are not observed at later stages. Its bipolar morphology and axial alignment (Figs. $2 B, 6 D$ ), its broad lamellae apposed to the inner epithelial surface (Fig. 1C), and its characteristic dorsal location suggest that it is one of a set of identified muscle pioneer cells in this region (Ball et al., 1985; Ho et al., 1983; Robert Ho, personal communication).

Filopodia regularly appear to be oriented specifically toward the $m l$ cell (Figs. $1 C, 6, C, D$ ). On occasion, multiple filopodia directly contact this cell (Fig. $6 D$ ). Furthermore, after the Til growth cones have reoriented and extended to the Trl cell, clusters of filopodia are regularly observed extending from the reorientation site toward the $m 1$ cell (Figs. 5, $A, C, 8$ ).

Other factors may also contribute to guidance along the $c$ segment. The $c$ segment does not always orient directly toward the $m l$ cell (Fig. 8). Another cell, $m 2$, occasionally labels in the same dorsal region as $m l$, and may contribute to growth cone guidance along segment $c$. The segment boundary between the femur and trochanter also develops near the termination point of the $c$ segment (Caudy and Bentley, 1986) and may also contribute to Til morphologies there. Furthermore, the distinct proximodorsal reorientation that initiates growth along the $c$ segment occurs before the growth cones appear to be in filopodial contact with the $m l$ cell or other dorsally located cells (Fig. 1B). Thus, there may be other, unknown cues involved in the initiation of growth along the $c$ segment. However, the $m 1$ cell frequently does appear to influence the Til growth cone in the terminal region of the $c$ segment (Figs. $6 D, 8$ ). We conclude that $m l$ contributes to branch extension and growth cone morphology on segment $c$, but that other factors may be involved.

\section{Segment e: ventral reorientation \\ from the $\operatorname{Tr} 1$ neuron}

On leaving the $\operatorname{Tr} 1$ guidepost neuron, the Til growth cones (Figs. 2B, $5 D$ ) and axons (Fig. 3; see also Bentley and Caudy, $1983 \mathrm{~b}$; and Caudy and Bentley, 1986) distinctly reorient circumferentially and extend ventrally to form the characteristic $e$ segment. The guidance cue for this segment of growth has been identified as a limb segment boundary (Bentley and Caudy, 1983b), in particular, the distal segment boundary of the coxa (Caudy and Bentley, 1986). This segment boundary differentiates within the epithelial substrate. Once on this continuous circumferential cue, growth cones apparently extend circumferentially along it until filopodial contact with the proximally located $\mathrm{Cx} 1$ neurons distinctly reorients them proximally (Fig. 3; Bentley and Caudy, 1983b).

\section{Segment g: proximal reorientation from the CX1 neurons}

On leaving the $\mathrm{Cx} 1$ guidepost neurons, the Til axons and growth cones orient proximally, along the $g$ segment. The $g$ segment characteristically is very straight, without lateral branches of either Til growth cones or axons (Fig. 4A).

Unlabeled axons from the $\mathrm{Cx} 1$ cells could provide a continuous cue for Til growth cones on the $g$ segment, since they sometimes pioneer the path to the CNS (Fig. 7). However, when the $\mathrm{Cxl}$ cells pioneer the pathway, they too are very highly oriented and do not branch (Fig. 7). The lack of lateral processes extends even to the lack of lateral filopodia (Fig. 7). One possible cue for guiding the Til and Cxl growth cones along the $g$ segment is a set of proximal cells, the " $p$ " cells, which appear to form a nearly continuous chain there (Fig. 4, $B, C, D$ ). These cells sporadically label with anti-HRP and may be present in all legs. Growth cones occasionally exhibit a low degree of fil- 
opodial wrapping of at least the most distal of these cells (Fig. $4 B$ ). However, growth cones (Fig. $7 A$ ) and axons (Figs. 4A, 7B) on the $g$ segment typically exhibit no branch or filopodial wrapping of cells there. We conclude that there is a strong extrinsic factor that orients growth cones on the $g$ segment and that the $p$ cells may sometimes contribute to guidance there.

\section{Normal variations in the axon path}

Seven characteristic axon segments in the overall Til path have been described. Different axon segments exhibit different degrees of variability in form and expression. The $g$ segment always is present, and always extends straight proximally from the Cx1 neurons. The $f$ segment also is always present, and always extends to the Cxl neurons. However, the point at which the $f$ segment begins can range from the point at the ventral edge of the Trl cells to any point more ventral along the distal segment boundary of the coxa, up to the point on the boundary that is straight distal to the $\mathrm{Cx} 1$ cells.

The $e$ segment is totally absent in pathways in which the Ti1 axons extend directly from the $\operatorname{Tr} 1$ neurons to the $\mathrm{Cx} 1$ neurons. When it is present, it always is oriented straight ventrally along the segment boundary and ranges in extension from the $\operatorname{Tr} 1$ neurons to the point straight distal to the Cx 1 neurons (Bentley and Caudy, 1983b; Caudy and Bentley, 1986).

The axon segments between the Til and Fel neurons can vary both in the exact direction of orientation, and in whether thcy arc present. The orientation of the $a$ segment can range over about $30^{\circ}$ of arc, between the line extending straight proximal along the geometric axis of the limb, and the line extending straight to the Fel neuron (schematized in Fig. 10, upper right). Axons occasionally extend straight from the Til neurons to the $\mathrm{Fel}$ guidepost neuron (Figs. $2 A, 5 B$ ). In such cases it is not possible to identify this straight segment as an $a$ segment or a $b$ segment, nor to determine if or where a switch in guidance cue was made. In other cases, the $b$ segment is definitely missing (Fig. 2B; see also Caudy and Bentley, 1986, fig. 2, $A, B$ ), and the Til axons do not directly contact a labeled Fel cell but pass dorsal to it.

Similarly, the axon segments between the Fel and Trl neurons can vary, both in whether they are present and in their exact orientation. Axons occasionally extend straight between these neurons, so that there is no apparent $c$ segment (Fig. 5, $C, D)$. However, even where there is no $c$ segment, filopodial extension often is biased dorsally (Figs. $5 C, 6 C$ ). In rare cases, axons can orient ventrally before reaching the distal coxa segment boundary and thus miss $\operatorname{Tr} 1$ (Fig. $4 C$; see also Caudy and Bentley, 1986); in these cases, both the $c$ and $d$ segments are missing.

The range of variation of axon pathways in normal embryos is schematized at the upper right of Figure 10. Other extreme variations occur in specially selected clutches of embryos where the Til growth cones extend proximally before the $\mathrm{Fe} 1$ and $\mathrm{Tr} 1$ guidepost neurons have differentiated (Caudy and Bentley, 1986). In such embryos, straight axon pathways from the Til neurons to the distal boundary of the coxa are regularly observed, and pathways frequently do not contact the $\mathrm{Fe} 1$ and $\operatorname{Tr} 1$ neurons once they differentiate (Caudy and Bentley, 1986, fig. 8F). Also, in these embryos growth cones in this region exhibit profuse branching and are not highly oriented (Caudy and Bentley, 1986).

\section{Orientations and reorientations of Til growth cones}

Growth cone morphologies confirm the above observations for axon segment orientations. Til growth cones in normal embryos have very few, if any, branches (Fig. 1). As a result, the orientation of the growth cone as a whole often can be clearly defined by the orientation of the axon or major branch trailing immediately behind the leading edge of the growth cone. Growth

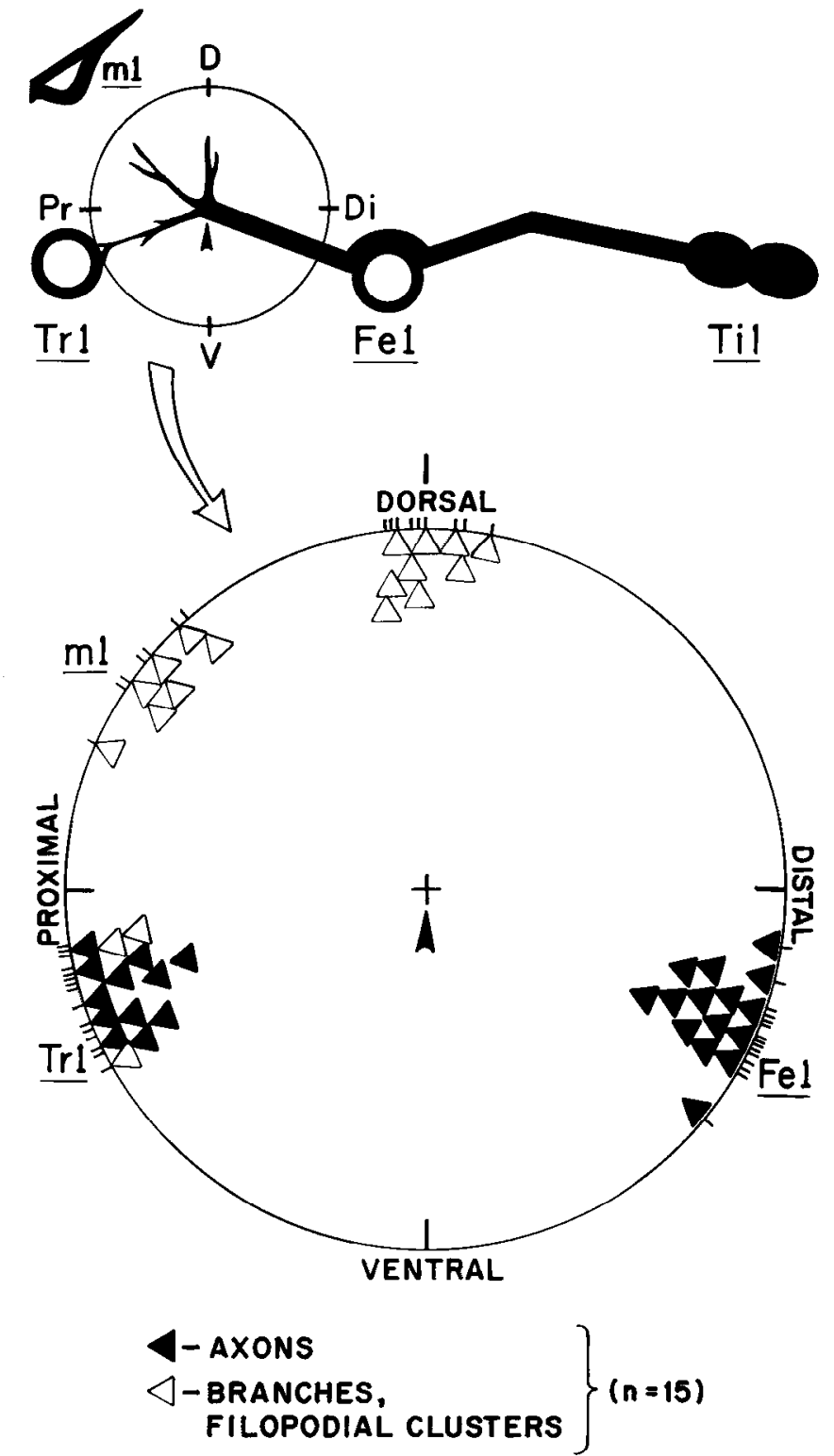

Figure 8. Characteristic Ti1 branches and filopodial clusters at the site of reorientation toward the $\operatorname{Tr} 1$ neurons. Upper diagram, Schematic representation of a Til neuron with axon segments along the $a$ and $b$ segments that has extended along the $c$ segment to the point (caret) of reorientation toward the one $\operatorname{Tr} l$ neuron. Branches are drawn in each of 3 directions characteristic of branch and filopodial cluster extension from that point: (1) directly toward the $\operatorname{Tr} 1$ neuron; (2) proximodorsally toward the $m l$ cell; and (3) straight dorsally from the reorientation point (possibly toward the $m 2$ cell, or along the prospective femur-trochanter segment boundary). Lower diagram, The reorientation site (carat) lies at the center of a polar plot of the directions of Til axons (solid triangles) and branches and filopodial clusters (open triangles) observed to extend from that point in 15 representative examples. Axons extended only to the $\operatorname{Tr} I$ cell, never in the other directions. Branches and filopodial clusters characteristically are oriented in the direction of the $m 1$ cell, and also straight dorsally. No branches or clusters are observed in other directions, nor do cells label in other directions. The inwardly oriented solid triangles, lower right, indicate the directions from which the Til axons approach the reorientation site along the $c$ segment. Note that these directions are frequently not oriented directly toward the $m l$ cell, although they may be in individual cases (e.g., Figs. $1 C, 4 B$ ). 


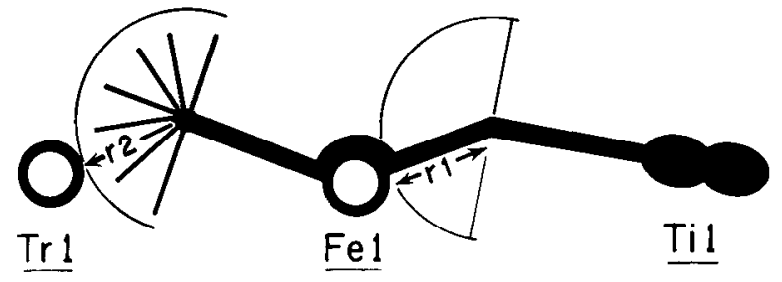

$$
\begin{aligned}
& r 1=33.8 \pm 8.5 \mu m(n=10) \\
& r 2=31.7 \pm 6.1 \mu m(n=11)
\end{aligned}
$$

Figure 9. Average distance at which $\mathrm{Til}$ axons reorient to the $\mathrm{Fel}$ and $\operatorname{Tr} 1$ neurons. The drawing schematizes a growth cone that has extended along the characteristic $a, b$, and $c$ segments and is at the reorientation site that initiates the $d$ segment that extends to the $\operatorname{Tr} l$ neuron. The rl and $\mathrm{r} 2$ distances are the average distances measured from the reorientation sites that initiate the $b$ and $d$ segments, respectively, to the point of contact with the Fel and $\operatorname{Tr} 1$ neurons.

cones at different locations along the path generally have the same orientation as do nascent axon segments observed at the same location in legs fixed at later stages. Thus, highly stereotyped orientations characterize both axons and growth cones at all points along the Til pathway.

\section{Disposition of growth cone branches}

Growth cone branches do not appear to be extended randomly in normal embryos. They characteristically extend around adjacent guidepost neurons or are oriented along the segments of the ultimate Til path (e.g., Figs. $1 C, 4 B, 5 A, 6, A, B$ ). Usually only single branches are oriented toward guidepost neurons (Figs. $1 C, 5 A$ ); multiple branches are sometimes observed oriented to other cues (Figs. $4 B, 6 B$ ). Branches that are extended lateral to the axon path are regularly observed at only 3 locations: (1) circumferentially at the distal segment boundary of the femur (Caudy and Bentley, 1986); (2) dorsally along the distal segment boundary of the coxa (Caudy and Bentley, 1986); and (3) at the site of reorientation that terminates the $c$ segment (Figs. $2 A, 8$ ). Branch and filopodial extension at segment boundaries is described elsewhere (Caudy and Bentley, 1986). We conclude that in normal embryos, growth cone branches are extended only toward or along specific extrinsic guidance cues.

\section{Disposition of growth cone flopodia}

Filopodia of Til growth cones fixed in situ are not disposed in a symmetrical fan at the leading edge of the growth cone, as is often seen in growth cone filopodia in culture (Bray, 1982; Letourneau, 1979). Rather, filopodia at the leading edge of Ti1 growth cones are predominantly (but not exclusively) oriented in the direction of extrinsic cues (Figs. 1, $B, C, 2 B, 3 B, 5, A, C$, $6, A, C, D, 7 A, 8)$. Single filopodia are regularly extended toward distant guidepost neurons in regions where there are no adjacent filopodia of comparable length (Fig. 1C). In addition, clusters of filopodia (defined in Materials and Methods) are predominantly oriented to specific cues, along the $c$ segment (Figs. 1, $B$, $C, 5 A, 6, A, C, 8$ ), along the $e$ segment (Fig. 2B), and along the $g$ segment (Fig. $7 A$ ). We conclude that the prominent filopodia observed to contact guidepost neurons and the distinct filopodial clusters oriented toward other cues reflect growth cone response to specific extrinsic cues.

\section{Growth cone reorientation process suggested by stereotyped Til morphologies}

The distinct reorientations of axon segments toward guidepost neurons suggest that growth cones also distinctly reorient toward them. Although the dynamic process of growth cone reorientation cannot be observed in fixed tissue, the stereotyped morphologies of Til growth cones suggest a particular sequence of growth cone response and reorientation to guidepost neurons.

Growth cones are observed that exhibit different degrees of orientation toward, and contact with, the $\operatorname{Tr} 1$ guidepost neurons: (1) growth cones oriented along the $c$ segment, which have no processes oriented toward the $\operatorname{Tr} 1$ neuron, although that cell has begun to label with anti-HRP and is therefore definitely present (Fig. $6 A$ ); (2) growth cones oriented along the $c$ segment, which have filopodia in direct contact with the Trl neuron but have no branches oriented toward that cell (not shown); (3) growth cones that have branches oriented directly toward the Trl neuron along a filopodium already in contact with it, although other growth cone branches and filopodial clusters also are present (Fig. 1C, $4 B$ ); (4) growth cones that have completely reoriented toward the $\operatorname{Tr} 1$ neuron, in that no branches are extended in other directions (Fig. 1D).

Similar observations have been made for growth cones that exhibit different degrees of orientation toward, and contact with, the $\mathrm{Cx} 1$ guidepost neurons: (1) growth cones that have oriented ventrally along the $e$ segment and have no processes oriented toward the Cx1 neurons (Figs. $2 B, 5 D$ ); (2) growth cones that have lateral filopodia in contact with the $\mathrm{Cx} 1$ neurons and also have branches extended further ventrally along the segment boundary cue for the $e$ segment (Fig. 3, $A, B$ ); (3) growth cones that have a branch oriented toward the $C_{x 1}$ neurons along filopodia in contact with them, although a branch is still further extended along the segment boundary (not shown here-see Bentley and Caudy, 1983b, fig. 4D); (4) growth cones that have completed reorientation toward the Cxl neurons, with no branches oriented further along the segment boundary (Fig. 3C).

The above observations suggest that growth cone branches are guided directly to guidepost neurons by extending along filopodia that have already made contact with those neurons. Furthermore, reorientations toward guidepost neurons are not observed in situations where the growth cones could not be in contact with an unlabeled guidepost cell. Together these observations also suggest that growth cones reorient by withdrawing branches extended in other directions and continuing to extend along branches oriented toward, and in filopodial contact with, guidepost neurons. Thus, the point from which a branch is extended toward a guidepost neuron apparently becomes the point of distinct, single reorientation observed between 2 adjacent axon segments at a later stage.

\section{Axon reorientations toward guidepost neurons} occur at characteristic distances

To determine whether growth cones generally could contact guidepost neurons with filopodia from the points where they reorient, we have measured the distance at which axons reorient toward the $\mathrm{Fe} 1$ and $\operatorname{Tr} 1$ guidepost neurons. The averages of these distance are the $r 1$ and $r 2$ distances depicted in Figure 9. On the average, Til growth cones reorient toward both the Fel and $\operatorname{Tr} 1$ neurons at a distance of about $30-35 \mu \mathrm{m}$. This distance often is spanned by Til filopodia (Figs. 1C, 3B). Thus, these results support the hypothesis that the Til growth cones contact distant guidepost neurons with filopodia, and then reorient directly toward them by extending branches along those filopodia.

\section{Discussion}

\section{Til morphologies reveal a sequence of distinct reorientations toward different cues}

The characteristic orientations of nascent axon segments are the clearest indication of guidance by a sequence of different cues (Figs. 1, 2, 3, 4A, 7B; schematized in Fig. 10). However, the same orientations also are evident in the leading ends of growth 


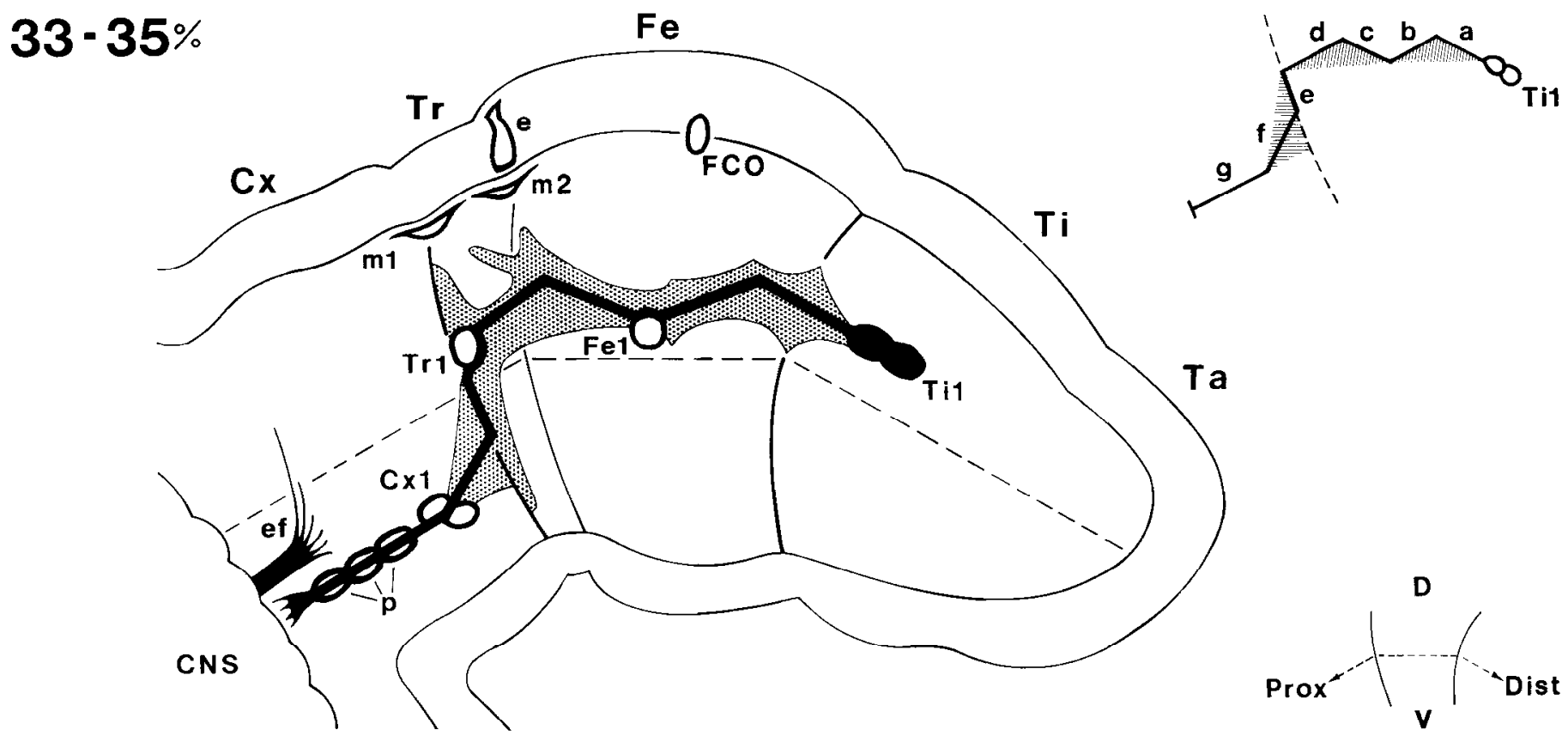

Figure 10. Summary diagram of the features of the leg and Til morphologies observed between the 33 and $35 \%$ stages. The pair of sibling TiI neurons have cell bodies that generally are aligned along the geometrical axis of the limb in the tibia (dashed line). The Ti1 growth cones emerge from the proximal poles of these cells and generally extend straight proximally along the limb axis, thus forming the characteristic $a$ segment. After about $50 \mu \mathrm{m}$ of proximal growth, the Til growth cones (in non-PPD clutches; $c f$. Caudy and Bentley, 1986) typically make a distinct reorientation in the proximoventral direction and extend directly toward and make contact with the $F e l$ guidepost neuron at the midpoint of the femur ( $F e$ ), thus establishing the $b$ segment. From the Fel cell, the Til growth cones typically orient proximodistally toward the region where the $m l$ cell labels occasionally, thus forming the $c$ axon segment. After extending in this direction, the Ti1 growth cones again distinctly reorient in the proximoventral direction and extend directly toward and make contact with the $\operatorname{Tr} 1$ guidepost neuron. This establishes the $d$ axon segment. After contacting the $\operatorname{Tr} 1$ neuron, the Til growth cones and axons reorient circumferentially and extend along the distal segment boundary of the coxa. The $e$ segment results from ventral growth along the segment boundary. The $f$ axon segment then is initiated by a distinct reorientation toward and direct extension to the $C x I$ guidepost neurons. After leaving the Cxl cells, the Til growth cones and axons extend proximally to the $C N S$, forming the $g$ axon segment. A chain of cells, the $p$ cells, sporadically and transiently labels with anti-HRP along the final path. The stippled region indicates the regions/ orientations in which Til branches normally occur. These include regions along the tibia-femur segment and trochanter-coxa segment boundaries (see Caudy and Bentley, 1986), as well as characteristic branches extended toward these $m l$ and also straight dorsally from the reorientation site between the $c$ and $d$ axon segments. Lateral branches are not observed on the $g$ segment. Til growth cones and axons sometimes extend directly between neurons on the pathway, and the Til axon morphology ranges between direct growth between these cells and the indirect, zigzag routes. This normal range of axon locations is indicated by the shaded regions adjacent to the axon segments depicted in the upper-right inset.

cones, as they extend along the path (Figs. 1, 2, 3C, 5A, 6, $A$, $B, 7 A$, and 8 ). Furthermore, these same orientations are observed for individual branches of growth cones, which appear to extend exclusively to specific cues (Figs. $1 C, 5 A, 8$ ). Finally, single filopodia (Figs. $1 C, 4 B$ ) and filopodial clusters (Figs. 1, $B, C, 2 B, 7 A, 8)$ also tend to orient in the direction of extrinsic cues.

Til growth cones that grow through the same region of the leg before specific cellular cues have differentiated do not exhibit the same characteristic orientation of growth cone processes (Caudy and Bentley, 1986). Thus, these specific orientations of Til processes are not due to intrinsic programs of neuronal morphogenesis but, rather, to response to extrinsic cues. (This also suggests that the distinct reorientations that have been analyzed here do not result from morphological deformation during tissue mounting.)

\section{Guidance cues for the Til pioneer growth cones}

The known and putative cues for the 7 characteristic axon segments are listed in Table 1 (see also Table 2). Cell-ablation experiments have shown the $\mathrm{Cx} 1$ neurons to be necessary and sufficient cues for guidance along the $f$ segment (Bentley and Caudy, 1983a, b). Til growth cones and axons typically exhibit the same specific interactions with the $\mathrm{Fe} 1$ and $\mathrm{Tr} 1$ neurons as they do with the Cxl guidepost neurons: (1) selective dye-cou- pling (Bentley and Keshishian, 1982a, b; Taghert et al., 1982); (2) selective branch and filopodial wrapping; and (3) characteristic reorientations toward, direct extension to, and further extension along those neurons. Thus, Til growth cones specifically interact with, and are guided by, each of the three guidepost neurons on the path.

Our results differ from those of Berlot and Goodman (1984), who concluded that the Fel neuron does not differentiate in time to influence the orientation or guidance of the Til growth cones. In contrast, we observe characteristic wrapping of branches and filopodia around the Fel neuron and, more importantly, the distinct reorientation of the Til growth cones and axons toward and along Fel. We conclude that in many embryos, the Fel (and Trl) neurons differentiate early enough to serve as guidepost neurons for the Ti1 growth cones and that in such embryos, the Til growth cones both respond to, and are guided by, those neurons (see also Bentley and Keshishian, 1982a, b; Keshishian and Bentley, 1983a).

There are cases, however, in which proximal axonogenesis can begin before filopodial contact is established with the Fel neuron (Bentley and Caudy, 1983b; Berlot and Goodman, 1984). In such cases, proximal outgrowth is along the characteristic $a$ segment and is oriented along the limb axis. Two possible guidance mechanisms for such proximal outgrowth have been suggested. The axial alignment of the Til cell bodies suggests that there may be an internal polarity to the Til neurons such that 
growth cones emerge from their proximal poles and continue proximally (Bentley and Caudy, 1983b). Altcrnativcly, there may be an extrinsic "limb axis cue," such as an adhesion gradient (Bentley and Caudy, 1983b; Berlot and Goodman, 1984). Evidence that the affinity of the epithelial substrate for growth cones increases proximally in this region has been presented elsewhere (Caudy and Bentley, 1986). Similar observations of directed outgrowth without guidepost cell contact have been made for pioneer axons in the wings of Drosophila (Blair and Palka, 1985; Blair et al., 1985; Schubiger and Palka, 1985).

\section{Til growth cones respond to cues of different cell types}

The Til growth cones respond to 3 different types of cells as they grow. The various guidepost cells are immature neurons. The guidance cue at the distal coxal segment boundary probably derives from (non-neuronal) epithelial cells, which are of ectodermal origin (Bentley and Caudy, 1983b; Caudy and Bentley, 1986). Finally, the $m I$ cell to which the growth cones respond as they extend along the $c$ segment is probably a muscle pioneer (Ball et al., 1985; Robert Ho, personal communication), and therefore of mesodermal origin.

\section{Hierarchy of affinities}

The Til pioneer growth cones exhibit a hierarchy of affinity for their various guidance cues. The Fel, $\operatorname{Tr} 1$, and $\mathrm{Cxl}$ guidepost neurons are the dominant cues in that once filopodial contact is established with a guidepost neuron, growth toward that neuron is completed before the Til growth cones reorient toward another cue.

Guidance along the $c$ segment may be provided by the $m l$ cell, and perhaps by additional cells in the dorsal region of the leg. Whether the $m l$ cell is the cue for the initial reorientation along the $c$ segment is uncertain. However, the Til growth cones definitely respond to it (Fig. 6D): They regularly extend branches and filopodial clusters dorsally well beyond the point at which the growth cones reorient to the $\operatorname{Tr} 1$ guidepost neuron (Fig. 8) and, in very rare cases, can continue to the dorsal edge of the leg if they do not reorient to $\mathrm{Trl}$ (Fig. 6B). Despite these specific responses, the Til growth cones normally reorient toward the $\operatorname{Tr} 1$ cell before they have completed growth to the $m l$ cell and other cells in the dorsal region (Fig. 1,C,D). It therefore seems probable that the $\operatorname{Trl}$ cell is a higher affinity cue than the $m l$ cell and other putative cues for the $c$ segment.

A similar inference can be made of the relative affinities of the distal coxal segment boundary and the $\mathrm{Cx} 1$ guidepost neurons. The segment boundary can provide a high-affinity cue for pioneer growth cones. Growth cones on the segment boundary can follow a very straight circumferential path along it for as much as $180^{\circ}$ of arc around the circumference of the leg (Bentley and Caudy, 1983b). However, Til axons always are observed to reorient away from the segment boundary and to extend directly to the Cx 1 neurons, although branches here also extend beyond the reorientation point, and growth on the segment boundary clearly could have continued (Caudy and Bentley, unpublished observations). Thus, the $\mathrm{Cx} 1$ neurons appear to be a higher affinity cue than the segment boundary.

The reorientation from the $a$ segment toward the Fel neuron may or may not reflect a hierarchy of affinity. Orientation along the $a$ segment may be due to an intrinsic mechanism rather than an extrinsic cue, in which case the reorientation toward $\mathrm{Fe} 1$ would not reflect a difference in affinities between extrinsic cues.

The reorientations that occur as growth cones leave a guidepost neuron do not reflect orientation along a cue of higher affinity, since no further guidance by that neuron is possible. The subsequent continuation of growth apparently reflects the absence of a specific "stop" signal for the Til growth cones, rather than reorientation along a higher affinity cue. Thus, for the Til growth concs, growth along a sequence of cues of different affinities does not require a continuous increase in affinity of cues in order for navigation to continue.

\section{Molecular aspects of the affinity hierarchy}

The differences in affinity exhibited by the Til growth concs for their various cues probably result from the differential expression of affinity molecules by them. There could be a difference in the degree of expression of a single type of affinity molecule on the different cues, or there could be different molecules for some or each of the cues. Also, these affinities could be adhesive in nature or could involve some active form of biochemical signaling (see also Caudy and Bentley, 1986). The differential expression of adhesion molecules on the cues might explain the observed preferential disposition of filopodia toward specific cues. If the cues are adhesive for filopodia, randomly extended filopodia would be preferentially retained at cues, while other filopodia are withdrawn (Bray, 1982, Letourneau, 1983). However, it is also possible that the various cues are adhesive for filopodia, but that some or all cues also interact actively. The selective establishment of dye-coupling with the guidepost neurons (Bentley and Keshishian, 1982a, b; Keshishian and Bentley, 1983a; Taghert et al., 1982), indicates that these cells, at least, may undergo more than simple adhesive interactions with pioncer growth cones.

Interestingly, cues of all 3 cell types label with anti-HRP, which generally is specific for surface molecules on neurons (Jan and Jan, 1982). Although the $m l$ cell apparently is a mesodermal cell, it still labels with anti-HRP. Similarly, the epithelial cells at segment boundaries also tend to label with anti-HRP (Caudy and Bentley, unpublished observations), and segment boundaries form high-affinity cues for the Til growth cones. Furthermore, the observed differences in affinity correlate with the degree of expression of the molecular binding site for anti-HRP. The highest affinity cues are the guidepost neurons, which all label strongly and consistently with anti-HRP by the $35 \%$ stage. The lower affinity cues such as the $m l$ cell and segment boundaries label weakly and inconsistently. Thus, the anti-HRP binding site might be a molecular cue for neuronal recognition, or it might be a surface molecule whose expression is linked to the expression of such a cue.

\section{Characteristics of growth cone steering}

\section{Growth cones steer by selective branch extension}

The process of growth cone reorientation toward guidepost neurons was inferred from Til morphologies consistently observed in fixed tissue (see Results). Growth cone branches are observed to extend along filopodia already in contact with guidepost neurons (Fig. 1C). The growth cone apparently reorients by extending along that branch and by withdrawing branches extended in other directions.

This process is supported by the additional observation that growth cone branches are not only directly extended to specific cues, but are also exclusively extended to cues (Fig. 8). Thus, the observed extension of a branch toward a guidepost neuron probably in no case is due to random growth. This strengthens the interpretation that branches are selectively extended along filopodia in contact with guidepost ncurons in responsc to that contact.

An analogous sequence of growth cone reorientation toward cues other than guidepost cells also can be inferred from stereotyped Til morphologies. For example, Til growth cones that have reached the $\mathrm{Fe} 1$ cell typically extend clusters of filopodia proximodorsally, along the $c$ segment (Fig. $1 B$ ). At slightly later stages, Til growth cone branches selectively extended along the $c$ segment are observed (Fig. 1C). At later stages, nascent axon 
segments are observed that are oriented along the $c$ segment (Fig. 1D)

Thus, both for reorientations toward guidepost neurons and for reorientations to other cues, a distinct reorientation in the nascent axon pathway is established by selectively extending a branch along, or to, the cue for the next segment. This selective extension of branches is in contrast to the extension of multiple, variously oriented branches in clutches of embryos in which the Til growth cones extend proximally before the Fel and $\operatorname{Tr} 1$ neurons and other specific cues have differentiated (see discussion of "PPD clutches" in Caudy and Bentley, 1986).

\section{Selective branch extension along oriented filopodia}

Filopodia in fixed tissue are not randomly oriented in advance of growth cone branches, but themselves tend to be oriented to the same cucs. The disposition of filopodia that precede branches varies widely, apparently depending on the nature of the cue being followed. Clusters of filopodia oriented directly ahead of branches are regularly observed on the $c$ segment (Figs. $1 C, 6 A$ ), on the $e$ segment (Fig. $2 B$ ), and on the $g$ segment (Fig. $7 A$ ). The width of the cluster varies, being very narrow on the $g$ segment and relatively wide on the $c$ and $e$ segments.

Filopodial dispositions on the segment boundary cue for the $e$ segment vary greatly, perhaps due to variation in the degree to which the boundary has differentiated at the time of growth along it. Filopodial clusters are regularly observed there (Fig. $2 B$ ), but branches sometimes extend along it without preceding filopodia (Fig. $5 D$ ). Growth cones also have been observed to orient along the segment boundary following a single thin process, either a single filopodium or a very thin branch (see Bentley and Caudy, 1983b, fig. $4 D$; see also Shankland, 1981, for a discussion of similar growth cone morphologies).

In some embryos, the Til growth cones extend proximally before the Fel and Trl guidepost neurons have differentiated, apparently in response to a proximal increase in the affinity of the epithelial substrate within segments (see discussion of "PPD clutches" in Caudy and Bentley, 1986). This cue appears to be distributed over the epithelial substrate, and in PPD clutches the Til filopodia appear to be more randomly oriented over the epithelium. In general, the presence and disposition of filopodia extended ahead of growth cone branches may depend on (1) whether growth is along a continuous cue (such as the segment boundary) or (2) is toward a distant cue (such as guidepost neurons), (3) the relative affinity of the cue, and (4) the degree to which the cue is localized.

\section{Distinct, single reorientations imply distinct switches in guidance cue}

The single, distinct reorientations observed between nascent axon segments suggest that a discrete switch was made between guidance cues. This interpretation is supported by the further observation that at earlier stages, growth cone branches not only are oriented specifically to cues, but are also oriented exclusively to cues. At no stage are branches extended in intermediate (noncue) directions. Thus, at no stage or location does the direction of an individual branch appear to reflect the sum influence of multiple cues. This further suggests that a growth cone reorients by making an abrupt "switch" or commitment to extension along the branch oriented to the highest affinity cue, rather than by a gradual reorientation as the growth cone progresses.

\section{Direct filopodial contact guides branch extension to guidepost neurons}

The extension of growth cone branches toward distant guidepost neurons apparently results from direct filopodial contact. The consistent observation of growth cones with different degrees of orientation toward, and contact with, the $\operatorname{Tr} 1$ and $\mathrm{Cx} 1$ guidepost neurons suggests a process by which $\mathrm{Til}$ growth cones reorient to guidepost ncurons (sec Results). Direct filopodial contact apparently triggers selective branch extension toward guidepost neurons, as well as the eventual withdrawal of other branches previously extended to other cues.

Several further observations support this hypothesis. Branchcs are not observed that are oriented in the direction of guidepost neurons but are clearly not, or could not be, in direct filopodial contact with them. In addition, the distinct reorientations observed between nascent axon segments toward the Fel and $\operatorname{Tr} 1$ guidepost neurons both occur at a distance of 30-35 $\mu \mathrm{m}$, which corresponds to the filopodial reach of the growth cone (Bentley and Caudy, 1983a, b; Bentley and Keshishian, 1982a, b; Keshishian and Bentley, 1983a; Taghert et al., 1982). The fact that reorientations toward both of these neurons occur at about the same distance suggests that the same guidance mechanism occurs in both cases. Furthermore, filopodial contact with the $\mathrm{Cx} 1$ neurons from growth cones on segment $e$ also is frequently observed at this distance (Fig. 3B).Therefore, direct filopodial contact is a likely mechanism for selective branch extension, and commitment to growth, to guidepost neurons.

\section{Single filopodia can direct growth cones to guidepost neurons}

At all locations on the path, branches tend to be oriented to specific cues (e.g., Fig. 8). Therefore, branches oriented in the direction of a guidepost neuron are probably selectively extended to some cue in that direction rather than randomly extended. The observation of direct contact by a single filopodium (Fig. 1C) suggests both that the neuron is the cue, and also that single filopodial contact mediates selective branch extension. (It also is possible that the initiation of branch extension requires multiple filopodial contact, but, if so, the additional filopodia must be withdrawn as the branch extends along a single filopodium.)

Til growth cones generally extend single branches toward guidepost neurons. These single branches typically are extended along single filopodia that are in direct contact with those neurons. Branches in fixed tissue are observed at several apparent stages of extension along single filopodia to guidepost neurons: (1) Filopodial contact is made before branch extension begins (Fig. $3 B$ ); (2) a branch is extended partway along a single filopodium that is in direct contact with a guidepost neuron (Fig. 1C); (3) a branch is extended completely to the site of a guidepost neuron (Fig. 5A); (4) the growth cone has reoriented and has reached the guidepost neuron (Fig. $1 D$ ).

The progressive development of a branch extended along a single filopodium also is suggested by the observation of extremely thin branches (Fig. $4 B$ ) extended to distant guidepost neurons, as well as much thicker branches at presumably later stages (Fig. 5A). Although branch extension to guidepost neurons may sometimes occur along multiple filopodia, single filopodial contact regularly, and perhaps typically, directs branch extension to them.

\section{Single fllopodia can reorient growth \\ cones in the presence of more prominent processes}

Growth cones reorient toward guidepost neurons along single filopodia, even though multiple filopodia, or even prominent branches, still are preferentially oriented to the previous cue. Although these more prominent processes are eventually withdrawn, and may be in the process of withdrawal at the time of reorientation, they are present throughout the reorientation process, and somctimes for substantial pcriods afterward. For cxample, the multiple filopodia and/or branches that are selectively oriented along the $c$ segment are not only still present after the Til growth cones have reached the Trl neuron, but 
sometimes also after they have even reached the CNS (Fig. 4, $A, D$ ). Thus, contact with a guidepost neuron by a single filopodium consistently will reorient a growth cone that is still oriented to the previous cue.

\section{Implications for models of growth cone steering}

How does single filopodial contact with a guidepost neuron lead to selective branch extension and commitment to growth along that specific filopodium while many other filopodia remain preferentially oriented to the previous cue? Two basic types of growth cone steering models have been proposed. In their most extreme form, "passive adhesion" models propose that contraction forces generated within filopodia cause the retraction of nonadherent filopodia and produce traction forces along adherent filopodia that "pull the growth cone across the substrate" (Gunderson and Park, 1984). In such models, each filopodium that is selectively retained by adhesive contacts would contribute to the overall motive force exerted on the growth cone, and the final direction of movement would be in the direction of the vector sum of such forces. In contrast, "active" models involve biochemical response to contact with guidance cues, perhaps by regulating cytoskeletal conformation (Cooper and Schliwa, 1985), which might amplify relatively small differences between cues.

A problem with passive adhesion models is that differences in adhesion may be too weak to steer growth cones, let alone steer them along single filopodia. Growth cones in culture can extend directionally on growth factor-conditioned substrates where the spatial differences in the adhesivity of the substrate are relatively small (about 20\%; Gunderson and Park, 1984), suggesting that other processes may be necessary to steer growth cones, or at least to amplify adhesion differences that are present (Gunderson and Park, 1984).

Aligned microfilaments have been observed at the bases of filopodia fixed in culture (Letourneau, 1979). Two processes have been suggested by which relatively small adhesion differences might lead to growth cones steering along such aligned microfilaments: (1) Traction forces along filopodia "may be directed to pull forward intracellular structures that interact with actin filaments" (Letourneau, 1979; see also Bray, 1979), rather than to pull the whole growth cone; or alternatively, (2) forces generated along actin filaments within filopodia "may be transmittcd to microtubules and other structures [within the growth cone] to determine their positions in the growth cone margin" (Letourneau, 1983). The directed orientation of microtubules could then produce directed branch extension by an active process of adding monomers to the microtubule ends. In this way an active process would amplify an initial response that was based on relatively weak differences in adhesion.

However, the same alignment that mediates these two processes could also arise from an active response to a nonadhesive cue. For example, a filopodium in contact with a guidepost neuron might send a second messenger down its length to its base, where it could, for example, cause a localized contraction of the actomyosin known to be present there. Pioneer growth cones selectively dye-couple with guidepost neurons (Bentley and Keshishian, 1982a, b; Keshishian and Bentley, 1983a; Taghert et al., 1982), and filopodia and neuronal cues also undergo other active interactions (Bastiani and Goodman, 1984). Thus, active responses to filopodial contact with guidepost neurons are a reasonable possibility.

Does differential adhesion mediate the steering response of the Til pioneer growth cones? Recall that multiple filopodia and/or branches are observed to be selectively oriented toward the $m l$ cell while the growth cone is in the process of reorientation to $\operatorname{Tr} \mathrm{l}$ and are often observed well after the completion of that reorientation. According to strictly passive adhesion models of steering, these processes should have been selectively retained by adhesive contacts. As a result, they also should exert traction forces on the growth cone that influence its direction of growth. However, no such influence is observed, nor do Til growth cones steer in the direction of the greatest number of selectively retained filopodia.

Thus, the Til growth cones do not appear to be steered by the vector sum of filopodial forces acting on the growth cone. The growth cone neither advances nor is it pulled as a unit along the epithelial substrate, since filopodial clusters and prominent branches remain at their original locations for significant periods of time after the growth cone reorients. Rather, the growth cone can respond independently to multiple cues, although it consistently reorients to the cue of highest affinity, and eventually withdraws branches previously extended to cues of lesser affinity.

Finally, growth cones also appear to make a distinct switch in the cue to which they are steering, and probably can do so on the basis of contact by a single filopodium. Our results are not consistent with a strictly passive adhesion model of growth cone steering, hut they are consistent with models of steering by differential adhesivity of cues in which intracellular events amplify adhesive interactions sufficiently for growth cones to reorient along single filopodia. Alternatively, the results are also consistent with purely active models of steering that do not involve adhesion differences between cues, but, rather, are mediated solely by active guidance molecules.

\section{References}

Ball, E. E., R. K. Ho, and C. S. Goodman (1985) Muscle development in the grasshopper embryo. I. Muscles, nerves, and apodemes in the metathoracic leg. Dev. Biol. 111: 383-398.

Bastiani, M. J., and C. S. Goodman (1984) Neuronal growth cones: Specific interactions mediated by filopodial insertions and induction of coated vesicles. Proc. Natl. Acad. Sci. USA 81: 1849-1853.

Bate, C. M. (1976) Pioneer neurons in an insect embryo. Nature 260: 54-56.

Bentley, D., and M. Caudy (1983a) Pioneer axons lose directed growth after selective killing of guidepost cells. Nature 304: 62-65.

Bentley, D., and M. Caudy (1983b) Navigational substrates for peripheral pioneer growth cones: Limb-axis polarity cues, limb-segment boundaries, and guidepost neurons. Cold Spring Harbor Symp. Quant. Biol. 48: 573-585.

Bentley, D., and H. Keshishian (1982a) Pioneer neurons and pathways in insect appendages. Trends Neurosci. 5: 364-367.

Bentley, D., and H. Keshishian (1982b) Pathfinding by peripheral pioneer neurons in grasshoppers. Science 218: 1082-1088.

Bentley, D., H. Keshishian, M. Shankland, and A. Toroian-Raymond (1979) Quantitative staging of embryonic development of the grasshopper, Schistocerca nitens. J. Embryol. Exp. Morphol. 54: 47-74.

Berlot, J., and C. S. Goodman (1984) Guidance of peripheral pioneer neurons in the grasshopper: Adhesive hierarchy of epithelial and neuronal surfaces. Science 223: 493-496.

Blair, S., and J. Palka (1985) Axon guidance in cultured wing discs and disc fragments of Drosophila. Dev. Biol. 108: 411-419.

Blair, S., M. A. Murray, and J. Palka (1985) Axon guidance in cultured epithelial fragments of the Drosophila wing. Nature 315: 406-408.

Bray, D. (1979) Mechanical tension produced by nerve cells in culture. J. Cell Sci. 37: 391-410.

Bray, D. (1982) Filopodial contraction and growth cone guidance. In Cell Behaviour: A Tribute to Michael Abercrombie, R. Bellairs et al., eds., pp. 299-317, Cambridge U. P., New York.

Caudy, M., and D. Bentley (1986) Pioneer growth cone morphologies reveal proximal increases in substrate affinity within leg segments of grasshopper embryos. J. Neurosci. 6: 364-379.

Cooper, M. S., and M. Schliwa (1985) Electrical and ionic control of tissue cell locomotion in DC electric fields. J. Neurosci. Res. 13: 223244.

Gunderson, R. W., and K. H. C. Park (1984) The effects of conditioned media on spinal neurites: Substrate-associated changes in neurite direction and adherence. Dev. Biol. 104: 18-27.

Ho, R. K., and C. S. Goodman (1982) Peripheral pathways are pioneered by an array of central and peripheral neurones in grasshopper embryos. Nature 297: 404-406. 
Ho, R. K., E. E. Ball, and C. S. Goodman (1983) Muscle pioneers: Large mesodermal cells that erect a scaffold for developing muscles and motoneurones in grasshopper embryos. Nature 301: 66 .

Jan, L. Y., and Y. N. Jan (1982) Antibodies to horseradish peroxidase as specific neuronal markers in Drosophila and grasshopper embryos. Proc. Natl. Acad. Sci. USA 79: 2700-2704.

Keshishian, H., and D. Bentley (1983a) Embryogenesis of peripheral nerve pathways in grasshopper legs. I. The initial nerve pathway to the CNS. Dev. Biol. 96: 89-102.

Keshishian, H., and D. Bentley (1983b) Embryogenesis of peripheral nerve pathways in grasshopper legs. II. The major nerve routes. Dev. Biol. 96: 103-115.

Keshishian, H., and D. Bentley (1983c) Embryogenesis of peripheral nerve pathways in grasshopper legs. III. Development without pioneer neurons. Dev. Biol. 96: 116-124.

Letourneau, P. C. (1975a) Possible roles for cell-to-substratum adhesion in neuronal morphogenesis. Dev. Biol. 44: 77-91.

Letourneau, P. C. (1975b) Cell-to-substratum adhesion and guidance of axonal elongation. Dev. Biol. 44: 92-102.
Letourneau, P. C. (1979) Cell-substratum adhesion of neurite growth cones and its role in neurite elongation. Exp. Cell. Res. 124: 127138.

Letourneau, P. C. (1983) Axonal growth and guidance. Trends Neurosci. $6: 451-455$.

Schubiger, M., and J. Palka (1985) Genetic suppression of putative guidepost cells: Effect on establishment of nerve pathways in Drosophila wings. Dev. Biol. 108: 399-410.

Shankland, M. (1981) Embryonic development of a sensory afferent projection in the grasshopper embryo. I. Growth of peripheral pioneer axons within the central nervous system. J. Embryol. Exp. Morphol. 64: $169-185$.

Shankland, M., and D. Bentley (1983) Sensory receptor differentiation and axonal pathfinding in the cercus of the grasshopper embryo. Dev. Biol. 97: 468-482.

Taghert, P. H., M. J. Bastiani, R. K. Ho, and C. S. Goodman (1982) Guidance of pioneer growth cones: Filopodial contacts and coupling revealed with an antibody to lucifer yellow. Dev. Biol. 94: 391-399. 